\title{
Effects of LHRF on toroidal rotation in Alcator C-Mod plasmas
}

\author{
J.E. Rice ${ }^{1}$, Y.A. Podpaly ${ }^{1}$, M.L. Reinke ${ }^{1}$, C. Gao ${ }^{1}$, S. Shiraiwa ${ }^{1}$, \\ J.L. Terry ${ }^{1}$, C. Theiler $^{1}$, G.M. Wallace ${ }^{1}$, P.T. Bonoli ${ }^{1}$, D. Brunner ${ }^{1}$, \\ R.M. Churchill ${ }^{1}$, I. Cziegler ${ }^{2}$, L. Delgado-Aparicio ${ }^{3}$, \\ P.H. Diamond ${ }^{2}$, I.C. Faust ${ }^{1}$, N.J. Fisch ${ }^{3}$, R.S. Granetz ${ }^{1}$, \\ M.J. Greenwald ${ }^{1}$, A.E. Hubbard ${ }^{1}$, J.W. Hughes ${ }^{1}$, \\ I.H. Hutchinson ${ }^{1}$, J.H. Irby ${ }^{1}$, J. Lee ${ }^{1}$, Y. Lin ${ }^{1}$, E.S. Marmar ${ }^{1}$, \\ R. Mumgaard ${ }^{1}$, R.R. Parker ${ }^{1}$, S.D. Scott $^{3}$, J.R. Walk ${ }^{1}$, \\ S.M. Wolfe ${ }^{1}$ and S.J. Wukitch ${ }^{1}$ \\ ${ }^{1}$ PSFC, MIT, Cambridge, MA 02139, USA \\ ${ }^{2}$ CMTFO, UCSD, San Diego, CA 92903, USA \\ ${ }^{3}$ PPPL, Princeton, NJ 08543, USA
}

Received 8 April 2013, accepted for publication 24 July 2013

Published 16 August 2013

Online at stacks.iop.org/NF/53/093015

\begin{abstract}
Application of lower hybrid range of frequencies (LHRF) waves can induce both co- and counter-current directed changes in toroidal rotation in Alcator C-Mod plasmas, depending on the target plasma current, electron density, confinement regime and magnetic shear. For ohmic L-mode discharges with good core LH wave absorption, and significant current drive at a fixed LH power near $0.8 \mathrm{MW}$, the interior $(r / a<0.5)$ rotation increments (on a time scale of order the current relaxation time) in the counter-current direction if $n_{\mathrm{e}}\left(10^{20} \mathrm{~m}^{-3}\right)>q_{95} / 11.5$, and in the co-current direction if $n_{\mathrm{e}}\left(10^{20} \mathrm{~m}^{-3}\right)<q_{95} / 11.5$. All discharges with co-current rotation changes have $q_{0}>1$, indicating a good correlation with driven current fraction, unifying the results observed on various tokamaks. For high density $\left(n_{\mathrm{e}} \geqslant 1.2 \times 10^{20} \mathrm{~m}^{-3}\right) \mathrm{L}$-mode target discharges, where core LH wave absorption is low, the rotation change is in the co-current direction, but evolves on a shorter momentum transport time scale, and is seen across the entire spatial profile. For H-mode target plasmas, both co- and counter-current direction increments have been observed with LHRF. The H-mode co-rotation is correlated with the pedestal temperature gradient, which itself is enhanced by the LH waves absorbed in the plasma periphery. The H-mode counter-rotation increment, a flattening of the peaked velocity profile in the core, is consistent with a reduction in the momentum pinch correlated with a steepening of the core density profile. Most of these rotation changes must be due to indirect transport effects of LH waves on various parameters, which modify the momentum flux.
\end{abstract}

(Some figures may appear in colour only in the online journal)

\section{Introduction}

The benefits of rotation and flow shear for tokamak performance are well known. Toroidal rotation is often provided externally through neutral beam injection, but in future devices, neutral beam torque will be low, and other methods for rotation drive may be necessary. One possibility is to take advantage of self-generated flow in enhanced confinement regimes [1], but this process relies on plasma performance, which makes for a complicated control knob. Another approach is to utilize radio frequency waves: ion cyclotron, electron cyclotron and lower hybrid (LH). Through asymmetric launching with respect to the toroidal direction, all of these waves have been used to generate toroidal current, for which the current drive mechanisms are well documented [2], but the accompanying changes in the toroidal rotation are not well understood. Ion cyclotron range of frequencies (ICRF) mode conversion flow drive has been demonstrated [3-6], but the details of the velocity generation process are still unclear. Regarding rotation in ICRF minority heated $[7,8]$ and electron cyclotron heating $(\mathrm{ECH})$ [9-11] discharges, the direct momentum input is expected to be low, and the observed rotation is probably generated intrinsically, through modification of the temperature/pressure profiles. Toroidal rotation changes due to $\mathrm{LH}$ waves have also been observed [8,12-18], with co-current increments seen in JT-60U, Tore Supra, JET, EAST and C-Mod, and countercurrent modifications in C-Mod. In plasmas with good core 
absorption of LH waves, the mechanism for rotation drive is uncertain. Candidates include direct momentum input from the waves [19] (calculated to be low [8]), electron orbit loss $[12,14]$, trapped electron pinch effects [20], resonant electron radial drift [21,22], and less direct causes such as the turbulent equipartition pinch [17] or modification of the $q$ profile. A challenge of these accounts is to explain the direction of the change in rotation. The main goal of this paper is to document the rotation changes in C-Mod lower hybrid current drive (LHCD) plasmas, and to understand what determines the rotation increment direction. Also of interest are the rotation drive mechanisms in plasmas without core accessibility of the LH waves, or with poor core absorption. In these cases the source of the rotation is due to alteration of edge plasma conditions, which then leads to core rotation changes following momentum transport [16,23].

This detailed study of rotation in lower hybrid range of frequencies (LHRF) plasmas has been performed on the Alcator C-Mod tokamak [24] (major radius $R=0.67 \mathrm{~m}$, typical minor radius of $0.21 \mathrm{~m}$ ). Operation was in both forward and reversed magnetic field/plasma current, and in the upper single null (USN) and lower single null (LSN) configurations, with deuterium working gas. Line-averaged electron densities were in the range from 0.25 to $3.5 \times 10^{20} \mathrm{~m}^{-3}$, on axis magnetic field between 3.7 and $6.9 \mathrm{~T}$ and with plasma currents between 0.3 and $1.1 \mathrm{MA}\left(10 \geqslant q_{95} \geqslant 3.2\right)$. All target plasmas were sawtoothing before application of LHCD power and discharges with other significant MHD activity have been excluded. Electron densities were determined from a two colour interferometer, fast electron temperature evolution using electron cyclotron emission and $Z_{\text {eff }}$ from visible bremsstrahlung [25]. Electron density and temperature profiles were provided by Thomson scattering [25]. Magnetic flux surfaces were generated using EFIT [26] and current density profiles were also calculated from EFIT, where possible constrained by motional Stark effect (MSE) measurements $[25,27,28]$. Density fluctuations in the periphery were monitored using gas puff imaging [25]. Edge velocity and ion temperature profiles were determined from charge exchange recombination spectroscopy of boron ions [25]. Core toroidal rotation and ion temperature profiles were measured with a high resolution imaging $\mathrm{x}$-ray spectrometer system $[29,30]$, viewing $\mathrm{H}$ - and He-like argon. In the core plasma, this is a very good proxy for the main ion rotation velocity, since good agreement is shown with the rotation determined from sawtooth pre- and post-cursors, when available. There is no evidence for core poloidal rotation in these discharges as views from the top and bottom of the plasma show identical Doppler shifts. The argon diamagnetic velocity is also negligible due a small $1 / Z$ and relatively flat argon density profiles. Unless otherwise noted, toroidal rotation velocities are chord averaged. The velocity calibration was achieved by running locked mode discharges, which are presumed to have zero rotation velocity over the whole profile. Throughout this paper, '+' velocity indicates co-current directed rotation, while '-' indicates counter-current.

LH waves were introduced from a launcher which consists of 60 waveguides capable of delivering up to $1.2 \mathrm{MW}$ of power at $4.6 \mathrm{GHz}$, with parallel index of refraction, $n_{\|}$, between 1.5 and 4 [31], and with wave momentum input in the countercurrent direction. These parameters are favourable for toroidal

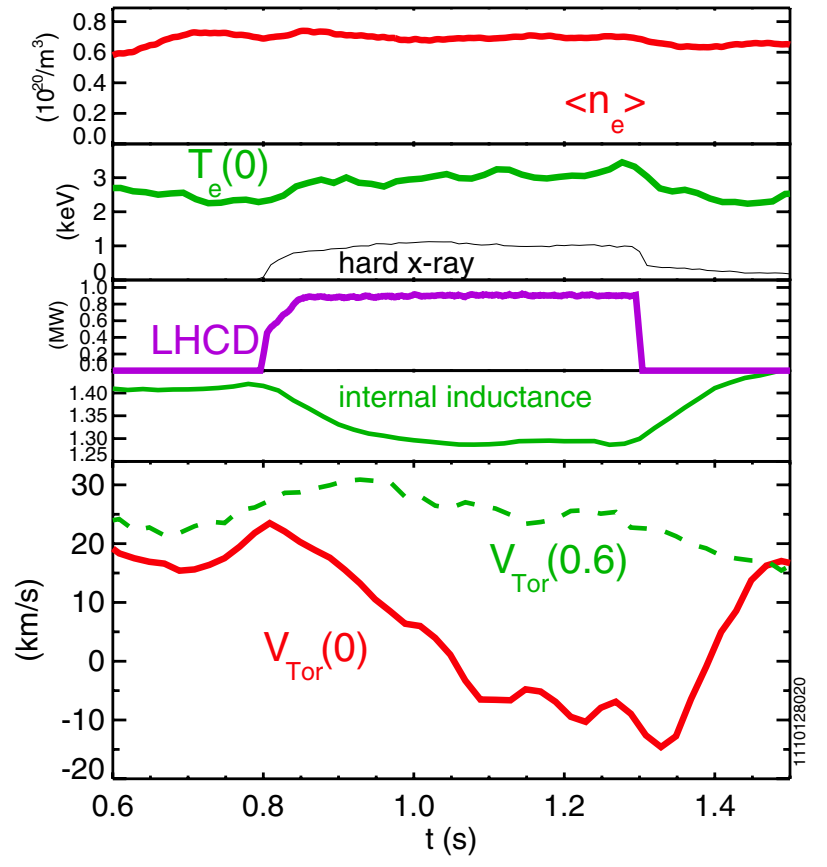

Figure 1. Time histories of the line-averaged electron density, central electron temperature (and core hard x-ray emission), $\mathrm{LH}$ power, internal inductance and toroidal rotation velocity from He-like Ar along two lines of sight (solid line through the plasma centre and dashed line tangent to $r / a=0.6)$ ) for a 5.4 T, 0.91 MA $\left(q_{95}=3.7\right)$ USN discharge.

electric current driven by LH waves, where the rf-driven current is generated efficiently in the co-current direction [32]. Recent results from C-Mod LHCD experiments may be found in [33-37]. H-mode is accessed with up to $5 \mathrm{MW}$ of ICRF power, usually by $\mathrm{H}$ minority heating in D plasmas [31] at $5.4 \mathrm{~T}$.

An outline of the paper follows. In section 2, velocity changes due to LHCD in low density ohmic L-mode target plasmas (with good core wave absorption) are presented, including spatial rotation profiles, in addition to scalings with plasma current, electron density and magnetic configuration. Velocity increments in both directions have been observed. A comparison of these results is made with ohmic rotation reversals in section 3. Rotation behaviour in higher density L-mode plasmas, with poor core $\mathrm{LH}$ wave absorption, is shown in section 4. Corresponding results to those presented in section 2 are described in section 5 for $\mathrm{H}$-mode target plasmas. Since these plasmas were at high density where the LH waves do not penetrate into the core plasma, rotation changes are due to modifications in the edge caused by the $\mathrm{LH}$ waves. A discussion of results is presented in section 6 , and various components of the momentum flux, which can be modified by LH waves, are examined.

\section{Velocity changes during LHCD in ohmic target discharges; plasma current and electron density dependence}

This section documents rotation changes in discharges with substantial core absorption of LH waves and significant current drive. Shown in figure 1 are the time histories of several parameters of interest for a $5.4 \mathrm{~T}, 0.91 \mathrm{MA}\left(q_{95}=3.7\right) \mathrm{USN}$ 


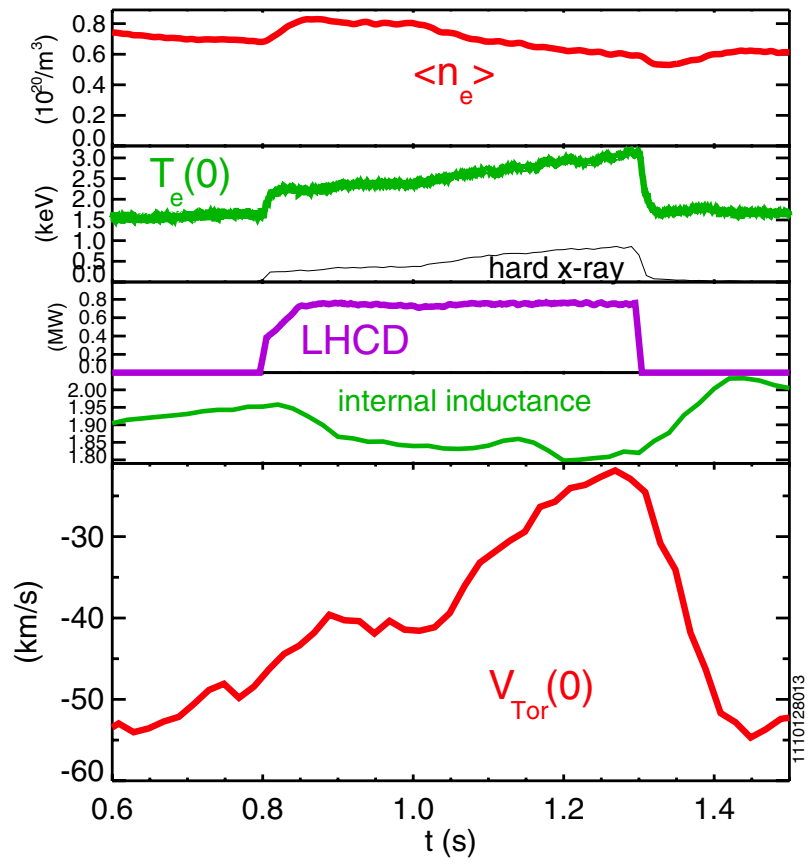

Figure 2. Time histories of the line-averaged electron density, central electron temperature (and core hard x-ray emission), $\mathrm{LH}$ power, internal inductance and central chord rotation velocity for a 5.4 T, $0.42 \mathrm{MA}\left(q_{95}=7.7\right)$ USN discharge.

deuterium discharge into which $0.9 \mathrm{MW}$ of LH power $\left(n_{\|}=\right.$ 1.6) were injected between 0.8 and $1.3 \mathrm{~s}$. While there was a modest increase in the electron temperature, the line-averaged electron density held constant at $0.7 \times 10^{20} \mathrm{~m}^{-3}$. There was a $10 \%$ drop in the internal inductance, $l_{\mathrm{i}}$, due to the $\mathrm{LH}$ power, indicating a broadening of the current density profile, which evolved on a time scale of several hundred $\mathrm{ms}$, of order the current relaxation time $(\sim 150 \mathrm{~ms})$, but in any case substantially longer than the global energy confinement time ( $\sim 30 \mathrm{~ms})$. There was also a decrease in the loop voltage, a slight rise in $q_{0}$ calculated from EFIT [26], and a substantial increase in core hard $\mathrm{x}$-ray emission. Estimated driven current was 0.5 MA out of 0.9 MA based on the loop voltage drop. There were sawtooth oscillations throughout the duration of the LHCD. There was a concomitant change in the core toroidal rotation velocity, incrementing in the counter-current sense from $\sim+20 \mathrm{~km} \mathrm{~s}^{-1}$ (co-current) to $\sim-10 \mathrm{~km} \mathrm{~s}^{-1}$ (counter-current), which also evolved on a time scale of $\sim 200 \mathrm{~ms}$; there was no velocity change outside of the mid-radius, confirmed by edge charge exchange measurements. This counter-current trend agrees with the wave momentum input direction and is consistent with previous C-Mod observations $[15,16]$ at a similar plasma current. Shown for comparison in figure 2 are the same traces for a $0.42 \mathrm{MA}\left(q_{95}=7.7\right)$ discharge with the same target density and similar LH power. While there was an analogous evolution of $l_{\mathrm{i}}$ and hard x-ray emission (and drop in the loop voltage) as in the higher current example, in this case the increment in the central chord rotation velocity was in the cocurrent direction, going from $\sim-50 \mathrm{~km} \mathrm{~s}^{-1}$ before application of the LH power to $\sim-25 \mathrm{~km} \mathrm{~s}^{-1}$ at the end of the LH wave injection. The rotation evolution is more complicated than that observed in figure 1. Compared to the plasma shown in figure 1, application of $\mathrm{LH}$ power drove a larger fraction of

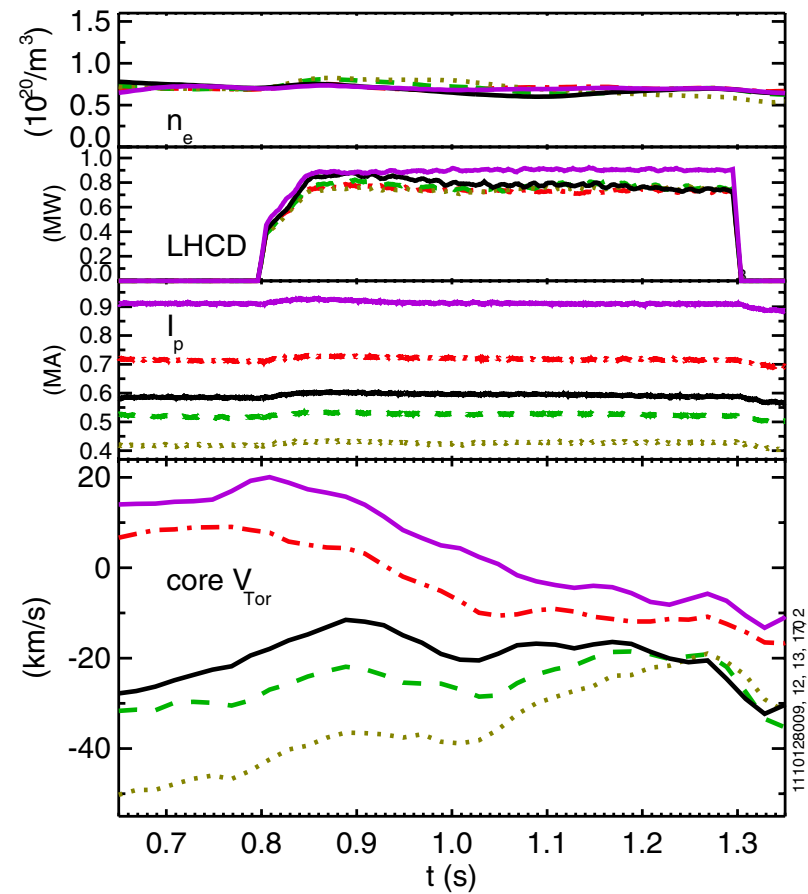

Figure 3. Time evolution comparison of the electron density, LH power, plasma current and core toroidal rotation velocity for five 5.4 T USN discharges with plasma currents of $0.91 \mathrm{MA}$ (dashed-dotted-dotted-dotted line), 0.71 MA (dashed-dotted), $0.58 \mathrm{MA}$ (solid), 0.52 MA (dashed) and 0.42 MA (dotted).

the total plasma current ( $0.3 \mathrm{MA}$ out of $0.42 \mathrm{MA})$ and led to a loss of sawteeth at $\sim 0.97 \mathrm{~s}$. This co-current increment of the toroidal rotation with LHCD is similar to previous observations from JT-60U [12, 14], Tore Supra [13], JET [8] and EAST [17].

It is important to understand what causes this bidirectional rotation with LHCD. This observed bi-directionality seems to rule out direct momentum input, since the waves are launched in the same direction in all cases. Interestingly, the input torque from LH waves $[8,19], R n_{\|} P_{\mathrm{LH}} / c \sim 0.002 \mathrm{~N} \mathrm{~m}$, is similar in magnitude to the torque required to accelerate the core plasma with density $\left\langle n_{\mathrm{e}}\right\rangle=0.7 \times 10^{20} \mathrm{~m}^{-3}$ by $30 \mathrm{~km} \mathrm{~s}^{-1}$ in $0.4 \mathrm{~s}$, about $0.003 \mathrm{Nm}$. This is a lower limit to the required torque, assuming long momentum confinement. To address this rotation increment direction issue, an examination of rotation characteristics has been performed during a comprehensive plasma current scan. A comparison of the core toroidal rotation velocity evolution following application of LH power for several different plasma currents, at fixed target electron density, in the USN configuration, is shown in figure 3 . The velocity increment is in the counter-current direction for higher target plasma current and in the co-current direction for the lower plasma currents. At the intermediate plasma current (0.58 MA), there is very little net change in the core rotation, which remains at $\sim-15 \mathrm{~km} \mathrm{~s}^{-1}$; this value serves as a velocity asymptote for the higher and lower current cases, at least for this LH input power. A counter-current offset has been observed in DIII-D magnetic braking experiments [38, 39]. The change/increment in the core rotation velocity (the difference in the time averaged velocity at the end of the LH pulse, $1.2-1.3 \mathrm{~s}$, and the pre-LH phase, $0.7-0.8 \mathrm{~s}$ ) as a function of plasma current, for a series of $5.4 \mathrm{~T}$ discharges with 


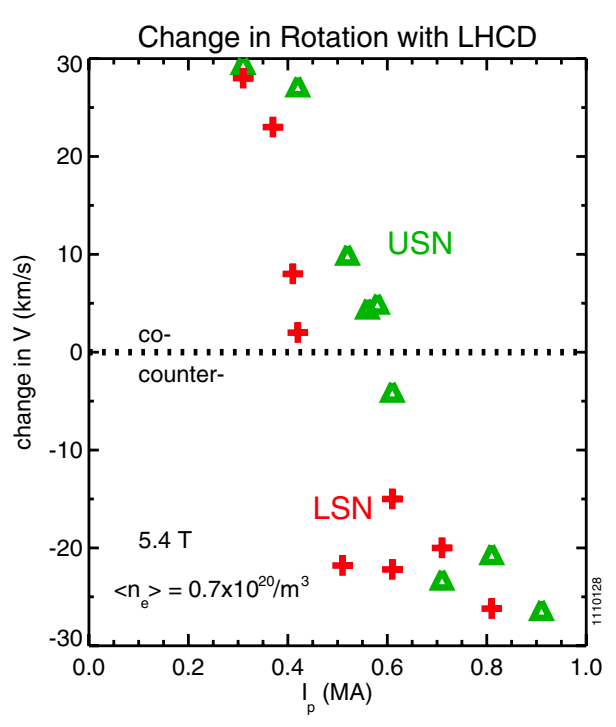

Figure 4. The change in the central chord averaged toroidal rotation velocity during LHCD $\left(0.75-0.9 \mathrm{MW}, n_{\|}=1.6\right)$ as a function of plasma current for 5.4 T, USN (triangles) and LSN (plus signs) discharges with $n_{\mathrm{e}}=0.7 \times 10^{20} \mathrm{~m}^{-3}$.

$n_{\mathrm{e}}=0.7 \times 10^{20} \mathrm{~m}^{-3}$ and $\mathrm{LH}$ power between 0.75 and $0.9 \mathrm{MW}$, is shown in figure 4 . The change in steady-state rotation varies strongly with plasma current, going from $\sim+30 \mathrm{~km} \mathrm{~s}^{-1}$ at the lowest current $\left(0.32 \mathrm{MA}, q_{95}=9.6\right)$ to around $-25 \mathrm{~km} \mathrm{~s}^{-1}$ at the highest current $\left(0.91 \mathrm{MA}, q_{95}=3.7\right)$. For the USN discharges (a subset of which is shown in figure 3), there is a stagnation point for $I_{\mathrm{P}} \sim 0.6 \mathrm{MA}\left(q_{95}=5.5\right)$ for these particular target plasma conditions, where application of $0.8 \mathrm{MW}$ of LH power has little effect on the central rotation. For the LSN plasmas, the stagnation point is at a lower plasma current, $\sim 0.45$ MA. The zero crossing point for the USN discharges is very close to the intrinsic rotation reversal critical density and current [40-42], suggesting a possible connection between this bi-directional rotation change with LHCD and intrinsic ohmic rotation reversals. The intrinsic rotation velocity of the ohmic target plasma also depends strongly on the plasma current $[8,42,43]$ as shown in figure 5 , from the same current scans, but before the LH wave injection. For the USN plasmas, the core toroidal rotation varies from $-60 \mathrm{~km} \mathrm{~s}^{-1}$ at the lowest $I_{\mathrm{P}}$ to $+20 \mathrm{~km} \mathrm{~s}^{-1}$ at the highest, and the intrinsic ohmic rotation switches direction at $\sim 0.7 \mathrm{MA}$ for this electron density and magnetic field, an example of the rotation reversal phenomenon [40-48]. The LSN points overlap at the lowest plasma currents, but then diverge as the current is raised above 0.6 MA. For this electron density, the LSN discharges do not exhibit a rotation reversal with increasing plasma current. A comparison of USN and LSN points for 0.8 MA indicates that the core rotation reverses with a change of magnetic configuration [46] due to changes in scrape-off layer (SOL) flows [49]. Reversals of intrinsic rotation with changes in the magnetic configuration have been observed in TCV plasmas [50]. It is interesting to note that even though the USN and LSN target plasmas at $0.8 \mathrm{MA}$ were rotating in opposite directions, the velocity increment with LHCD was counter-current in both cases. The LHRF wave injection shown in figures $1-5$ was with $n_{\|}=1.6$. The actual value of $n_{\|}$has little effect on the magnitude of the rotation change [16]. Furthermore, there is

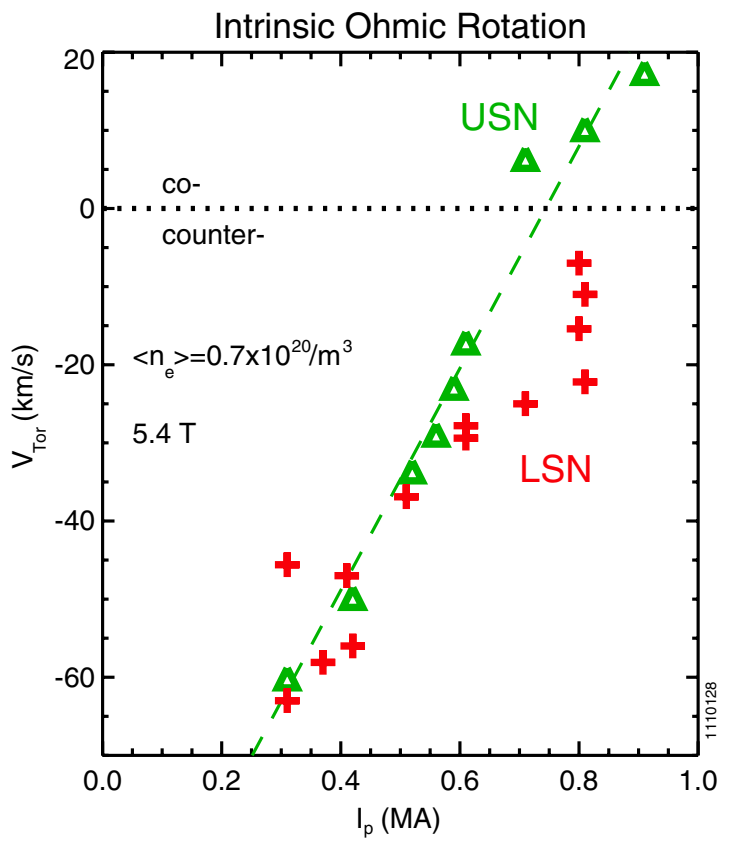

Figure 5. The core toroidal rotation velocity as a function of plasma current for 5.4 T, USN (triangles) and LSN (plus signs) discharges with $n_{\mathrm{e}}=0.7 \times 10^{20} \mathrm{~m}^{-3}$, during the ohmic phase before LHCD.

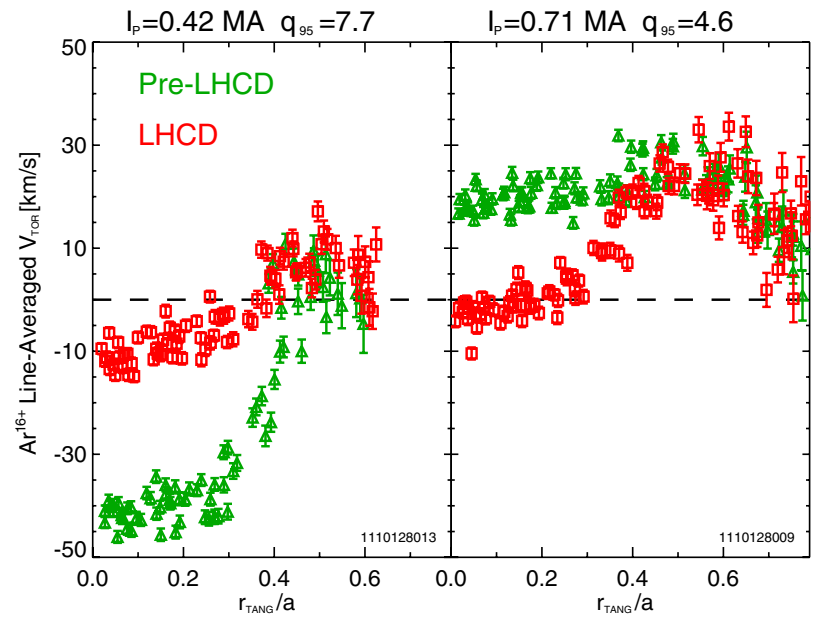

Figure 6. Toroidal rotation spatial profiles before (green triangles) and during (red boxes) LHCD for 0.42 MA (left) and 0.71 MA (right) discharges.

no observable difference in the sawtooth inversion radii for USN and LSN discharges at the same plasma current and density, for either the ohmic target phase (figure 5) or with LHCD (figure 4).

The results of figures 1-5 documented the core toroidal rotation velocity. Complete velocity profiles for high and low plasma current USN discharges before and during LHCD are shown in figure 6. For the 0.71 MA ohmic target plasma, the profile is relatively flat, with a co-current velocity around $+20 \mathrm{~km} \mathrm{~s}^{-1}$ across the profile. For the $0.42 \mathrm{MA}$ ohmic target plasma, the profile is strongly counter-current in the core and has a steep gradient region just inside of the mid-radius. During the LH wave injection, the profiles are similar, slightly countercurrent in the core. These particular shape changes suggest that these profiles are not the result of a momentum pinch 


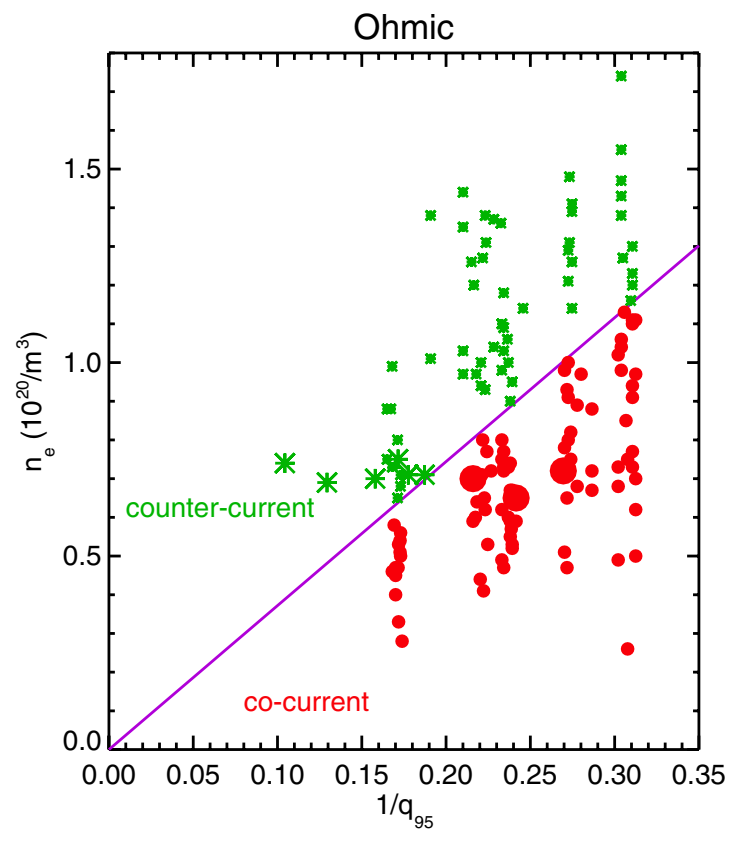

Figure 7. Operational ranges in the $n_{\mathrm{e}}-1 / q_{95}$ plane for ohmic discharges. Dots represent plasmas with co-current rotation while asterisks are for counter-current rotating discharges. The line indicates $n_{\mathrm{e}} q_{95}=3.7$, with $n_{\mathrm{e}}$ in units of $10^{20} \mathrm{~m}^{-3}$.

effect. There is no change in the profiles outside of the midradius $(r / a \sim 0.55)$ with $\operatorname{LHCD}[15,16]$. This anchoring of the profiles in the vicinity of the mid-radius is reminiscent of what is observed during ohmic rotation reversal [40]. This rotation profile anchoring and the similarity between the LHCD rotation bi-directionality and intrinsic ohmic rotation reversal critical points suggests that the two phenomena may be related.

\section{Comparison to ohmic rotation reversals}

The conjecture of a connexion between the ohmic rotation reversal process and the rotation direction changes in LHCD plasmas will be explored in this section. The intrinsic ohmic rotation reversal phenomenon is summarized in figure 7 , where the extended operating space with line-averaged density and $1 / q_{95}$ is shown (combination of USN and LSN discharges). Discharges rotating in the co-current direction are shown as dots while those rotating counter-current are depicted as asterisks. There is a very sharp boundary between intrinsic coand counter-rotating plasmas in the $n_{\mathrm{e}}-1 / q_{95}$ plane, which can be parametrized as $n_{\mathrm{e}} q_{95}=3.7$ [40, 42], the ohmic rotation reversal condition. The USN points from figure 5 are shown with the larger symbols.

The cause of this flip in rotation direction is thought to be due to a reversal in sign of the residual stress [51], $\Pi_{r}$, due to a change of domination from trapped electron modes (TEMs) to ion temperature gradient (ITG) modes above a critical collisionality $\left(v_{*}\right)$ [40-42]. $\left(\Pi_{r}\right.$ is the component of the momentum flux not proportional to the velocity or its gradient, whose sign depends on the underlying modes. The divergence of $\Pi_{r}$ is the intrinsic torque density.) This is why the co/counter boundary depends on the product $n_{\mathrm{e}} q_{95} \propto v_{*}$.

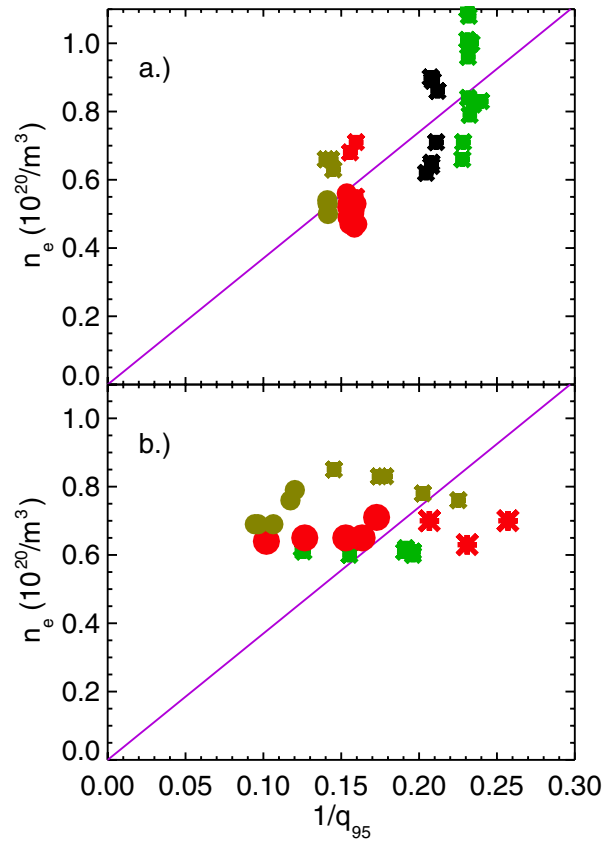

Figure 8. Operating points in the $n_{\mathrm{e}}-1 / q_{95}$ plane for LHCD plasmas. Dots indicate an increment in the co-current direction with LHCD while asterisks are for changes with LHCD in the counter-current direction. In the top frame $(a)$, different shades (colours) are from density scans at fixed current while in the bottom frame $(b)$, current scans at fixed density are shown. The lines indicates $n_{\mathrm{e}} q_{95}=3.7$, the ohmic intrinsic rotation reversal boundary, with $n_{\mathrm{e}}$ in units of $10^{20} \mathrm{~m}^{-3}$.

$\Pi_{r}$ also is a function of the gradient of the current density profile [51-55], and in principle can switch sign through changes in the current density profile by externally driven current via LH waves. Related plots for the operational space in LHCD plasmas from selected scans at fixed power are shown in figure 8, to be compared with figure 7. Asterisks depict a change between the ohmic target and LHCD rotation in the counter-current direction while dots are for increments in the co-current direction. The top panel is from dedicated density scans at fixed plasma current while the bottom frame shows the results from current scans at fixed density. The larger points in the bottom frame are from the current scan shown in figure 4. Different shades/colours are from selected scans. In some scans the switch between co- and counter-current direction increments with LHCD occurs at the ohmic rotation reversal critical boundary, while others clearly do not. Also, co- and counter-increments with LHCD appear on both sides of the boundary. Compare the $I_{\mathrm{P}}$ scan points from figure 4, shown by the larger red symbols in the lower panel, which are for USN plasmas, to the smaller mustard points just slightly above in density, which are for LSN discharges. Since the increment reversal points are different, this suggests that the magnetic configuration can affect the co/counter increment boundary, possibly through the $q$ profile. An up/down asymmetry in shape is also a possibility. It should be emphasized that in the LHCD cases, the symbols represent the change in rotation direction, while in the ohmic examples from figure 7, the actual rotation direction is shown. A similar plot from the complete LHRF database in L-mode target plasmas, including both USN and LSN plasmas, with power between 0.6 and $0.9 \mathrm{MW}$, and 


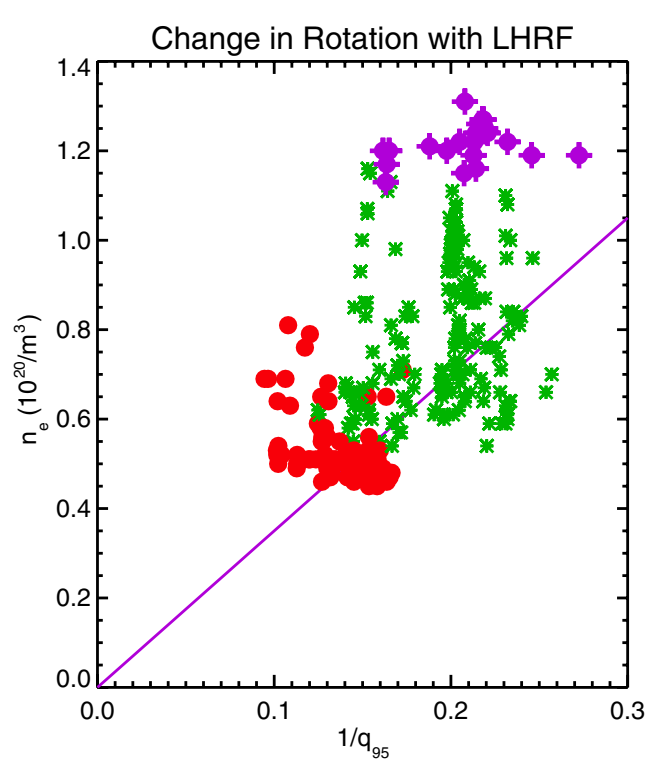

Figure 9. Operating points in the $n_{\mathrm{e}}-1 / q_{95}$ plane for L-mode LHRF plasmas. Red dots indicate a change in the co-current direction with LHCD while green asterisks are for changes with LHCD in the counter-current direction. The purple points (dots with $+\mathrm{s}$ ) are from high density discharges with poor core LH wave absorption. The line represents $n_{\mathrm{e}} q_{95}=3.7$, with $n_{\mathrm{e}}$ in units of $10^{20} \mathrm{~m}^{-3}$.

with $n_{\|}$between 1.6 and 2.5, is shown in figure 9. It is clear that there is nothing special about the ohmic rotation reversal boundary (solid line) and the co/counter rotation increment with LHCD. Inspection of this figure indicates that co-current increments with LHCD occur for low density, low current plasmas, which is consistent with the results from JT-60U, Tore Supra, JET and EAST. There is a group of points in figure 9 (represented by purple dots with ' + ' signs) indicating a cocurrent change with LHRF at the highest densities. These discharges, with low core LH wave absorption, will be treated in detail in the next section.

The clustering of LHCD co-current increment points at low density and current suggests that an alternative operational space plot would be more revealing. Shown in figure 10 is the corresponding graph in the $n_{\mathrm{e}}-q_{95}$ plane for LHCD rotation changes. There is a rough boundary between coand counter-increments with LHCD, represented by the line $n_{\mathrm{e}} / q_{95}=1 / 11.5=0.087$ (with $n_{\mathrm{e}}$ in units of $10^{20} \mathrm{~m}^{-3}$ ). There is a co-current change in most LHCD plasmas if $n_{\mathrm{e}, 20}<$ $q_{95} / 11.5$. This boundary is not as precise as the ohmic reversal boundary (figure 7) suggesting that there may be other parameter dependences (besides on $n_{\mathrm{e}}$ and $q_{95}$ ), such as on the current density profile or the magnetic configuration. For comparison, the ohmic rotation reversal boundary, $n_{\mathrm{e}} q_{95}=$ 3.7, is shown by the dashed line. (Again, the high density co-current points will be discussed in the next section.) Also shown are operational points from JT-60U, Tore Supra, JET and EAST LHCD plasmas, consistent with the C-Mod results, with co-current rotation at low density and low plasma current. However, it's not clear what the proper normalization for the electron density should be to get the most relevant comparison with observations from other devices. A summary of the LHCD rotation parameters from various tokamaks is shown in

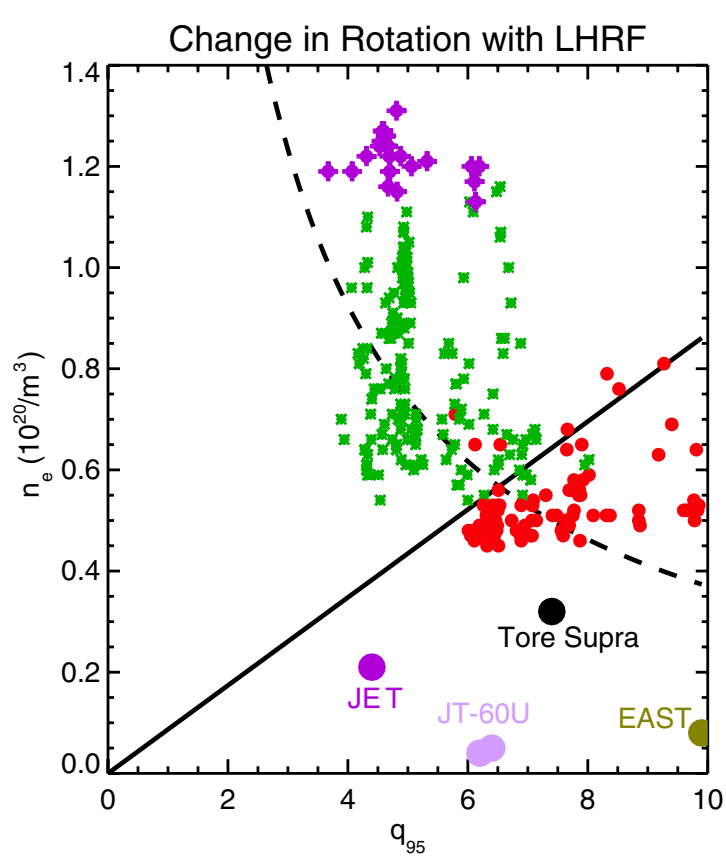

Figure 10. Operating points in the $n_{\mathrm{e}}-q_{95}$ plane for L-mode LHCD plasmas. Dots indicate an increment in the co-current direction with LHCD while asterisks are for changes with LHCD in the counter-current direction. The solid line represents $n_{\mathrm{e}} / q_{95}=1 / 11.5$, with $n_{\mathrm{e}}$ in units of $10^{20} \mathrm{~m}^{-3}$, and the dashed line is the ohmic rotation reversal boundary, $n_{\mathrm{e}} q_{95}=3.7$. Operating points for JT-60U (pink) [12], Tore Supra (black) [13], JET (purple) [8] and EAST (mustard) [17] are also shown.

table 1. The first column gives the rotation increment direction following LHCD.

Another way of approaching the comparison between ohmic rotation reversals and LHRF-induced rotation is to examine the rotation velocity as a function of the product of $n_{\mathrm{e}}$ and $q_{95}$. The product $n_{\mathrm{e}} q_{95}$ serves as a proxy for the collisionality. The result for a large number of ohmic plasmas is shown in figure 11. For $n_{\mathrm{e}} q_{95}<3.7$, the intrinsic rotation is directed co-current, and abruptly switches to counter-current for $n_{\mathrm{e}} q_{95}>3.7$ [40,42]. The ranges for $n_{\mathrm{e}}$ and $q_{95}$ in this plot are from 0.3 to $2.0 \times 10^{20} \mathrm{~m}^{-3}$ and 2.6 to 7.2 , respectively, with both USN and LSN. A related figure for LHRF plasmas is shown in figure 12 , where the change in rotation velocity is plotted as a function of the $n_{\mathrm{e}} q_{95}$ product. These discharges are sorted by 'favourable' and 'unfavourable' drift. 'Favourable' drift indicates that the ion $B \times \nabla B$ drift direction is towards the X-point. There seems to be no clear boundary in this plot, in contrast to the ohmic cases, which showed organization by collisionality. One thing that stands out from inspection of this figure is that most of the co-current increment discharges are with unfavourable drift (counter-current with favourable drift). However, this dataset may be biased since most of the unfavourable drift points were obtained with low density and high $q_{95}$, conducive to good current drive fraction, while most of the favourable drift points were from high density and low $q_{95}$ discharges. The edge cryopump was used to obtain the lowest density discharges; since the cryopump is located in the top of the vacuum chamber, it was necessary to run with USN, and hence unfavourable drift with the normal field direction. There seems to be no dependence on reversed field, and those 
Table 1. Machine and operational parameters for LHCD experiments in L-mode plasmas on various devices. The column marked $\Delta V$ indicates whether the change in rotation with LHCD was in the co- or counter-current (cntr) direction.

\begin{tabular}{lllllllll}
\hline Device & $\Delta V$ & $n_{\mathrm{e}}\left(10^{20} \mathrm{~m}^{-3}\right)$ & $q_{95}$ & $n_{\mathrm{e}} / q_{95}$ & $I(\mathrm{MA})$ & $B(\mathrm{~T})$ & $R(\mathrm{~m})$ & Ref. \\
\hline C-Mod & cntr & $0.6-1.2$ & $<5$ & $>0.087$ & 0.8 & 5.4 & 0.67 & {$[15,16]$} \\
C-Mod & co & $0.4-0.6$ & $6-10$ & $<0.087$ & $<0.6$ & 5.4 & 0.67 & {$[18]$} \\
EAST & co & 0.08 & 10 & 0.008 & 0.25 & 2 & 1.8 & {$[17]$} \\
JET & co & 0.21 & 4.4 & 0.048 & 2 & 2.6 & 2.96 & {$[8]$} \\
JT-60U & co & 0.05 & 6.4 & 0.008 & 1.2 & 4 & 3.4 & {$[12]$} \\
JT-60U & co & 0.04 & 6.2 & 0.006 & 1.2 & 3.6 & 3.4 & {$[14]$} \\
Tore Supra & co & 0.32 & 7.4 & 0.041 & 0.8 & 3.7 & 2.34 & {$[13]$} \\
\hline
\end{tabular}

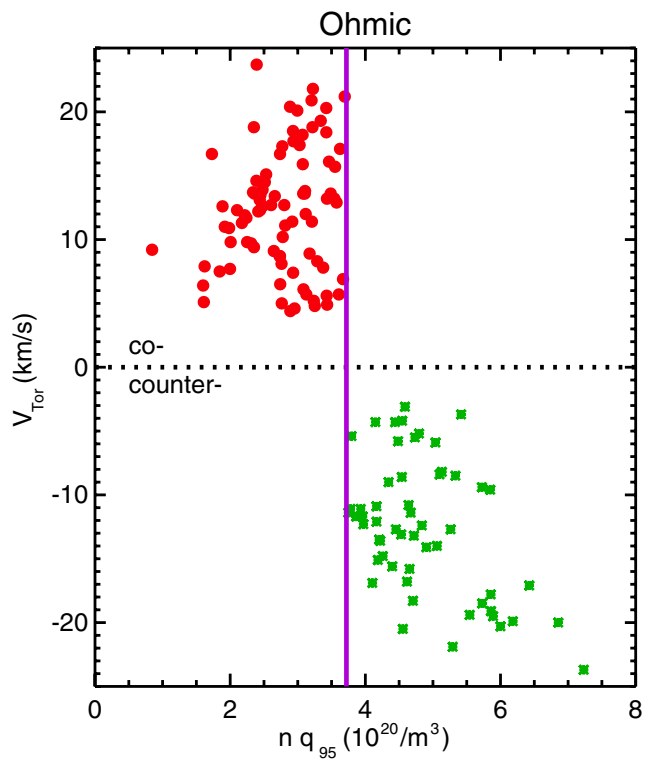

Figure 11. The toroidal rotation velocity as a function of the $n_{\mathrm{e}} q_{95}$ product for ohmic plasmas. The vertical line is $n_{\mathrm{e}} q_{95}=3.7$.

points (shown in lighter shades) are intermixed. It should be noted that when the magnetic field was reversed, the plasma current direction was also reversed. The product $n_{\mathrm{e}} q_{95}$ has the same density and plasma current dependence as the ratio $n_{\mathrm{e}} / n_{\mathrm{G}}$, where $n_{\mathrm{G}} \equiv I / \pi a^{2}$ is the density limit in $10^{20} \mathrm{~m}^{-3}$, with $I$ in MA and $a$ in $\mathrm{m}$. For the sake of completeness, the data in figure 12 are shown in figure 13, where the change in rotation velocity for LHRF plasmas is shown as a function of $n_{\mathrm{e}} / n_{\mathrm{G}}$. The scatter is a little larger, but the trends are the same as in figure 12.

Application of $\mathrm{LH}$ power can give rise to slight perturbations in the electron density, as can be seen in figures 1 and 2. If the target plasma is very close to the intrinsic ohmic rotation reversal boundary, this slight change in the density can lead to a reversal (the reversal boundary in figure 7 is very sharp), which can obscure the effects of LHRF on the rotation. An example is shown in figure 14, from a $5.4 \mathrm{~T}$, $0.55 \mathrm{MA}\left(q_{95}=6.0\right) \mathrm{LSN}$ discharge. Immediately following the $\mathrm{LH}$ power injection, there was a $10 \%$ increase in the electron density and a drop (and eventual reversal) in the cocurrent rotation, which evolved on a transport time scale (tens of ms), much faster than the velocity changes shown in figures 1 and 2. After $1.0 \mathrm{~s}$, the density began to decrease and the rotation returned to the co-current direction. This evolution is very similar to the rotation reversals shown in [41]. To complicate matters further, at $1.2 \mathrm{~s}$ there was an onset of MHD

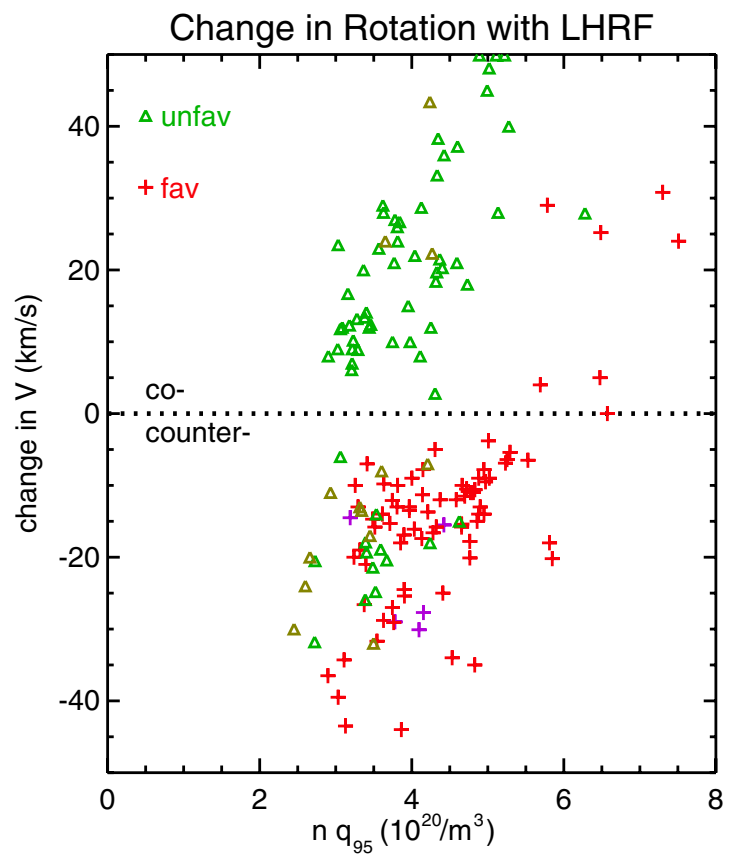

Figure 12. The change in the core toroidal rotation due to LHRF as a function of $n_{\mathrm{e}} q_{95}$. Triangles are for the unfavourable drift while plus signs are for favourable. Lighter shading indicates reversed magnetic field/plasma current.

activity, which led to a drop in the plasma stored energy, an increase in the internal inductance and a loss of current drive. Furthermore, many of these ohmic L-mode target plasmas, at low density and high current, are close to the threshold for locked mode formation [58,59]. Locked modes or other deleterious MHD activity can cause a braking of the core toroidal rotation, which in some cases can obscure the effects of LH waves [16]. Discharges with such significant MHD activity, and with complicated velocity evolution (figure 14), have been excluded in all scaling studies here.

\section{High density L-mode LHRF discharges}

There was a group of points in figures 9 and 10 with $n_{\mathrm{e}} \sim$ $1.2 \times 10^{20} \mathrm{~m}^{-3}$, and low core absorption of the LH waves, which had co-current rotation increments with LHRF power application. The rotation characteristics (evolution and spatial changes) in these discharges also exhibited much different behaviour than presented in section 2. Shown in figure 15 are the parameter time histories of one of these discharges, and two differences immediately stand out. The velocity 


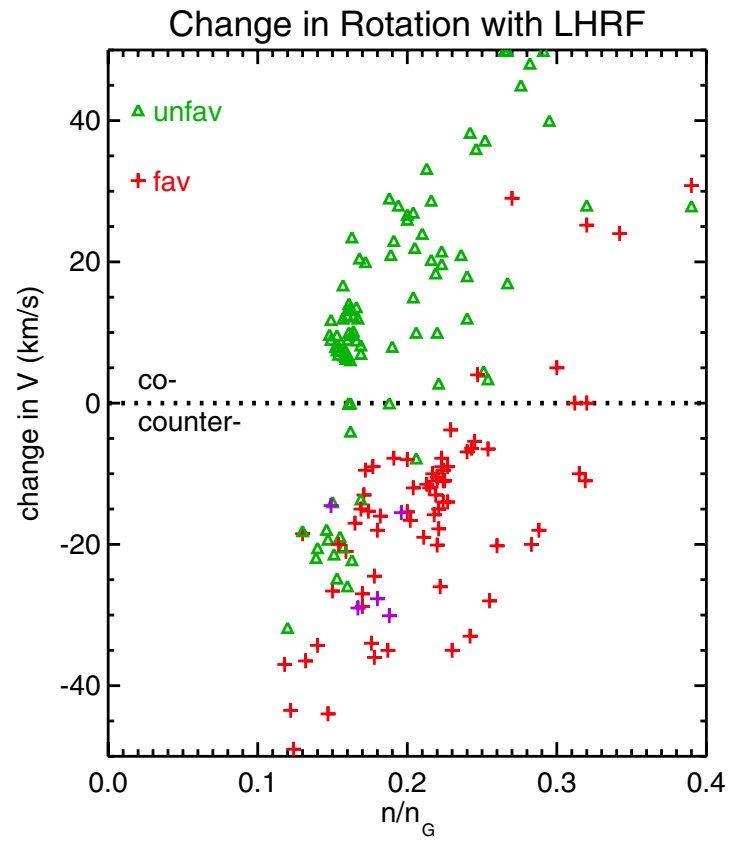

Figure 13. The change in the core toroidal rotation due to LHRF as a function of $n_{\mathrm{e}} / n_{\mathrm{G}}$. Triangles are for the unfavourable drift while plus signs are for favourable. Lighter shading indicates reversed magnetic field/plasma current.

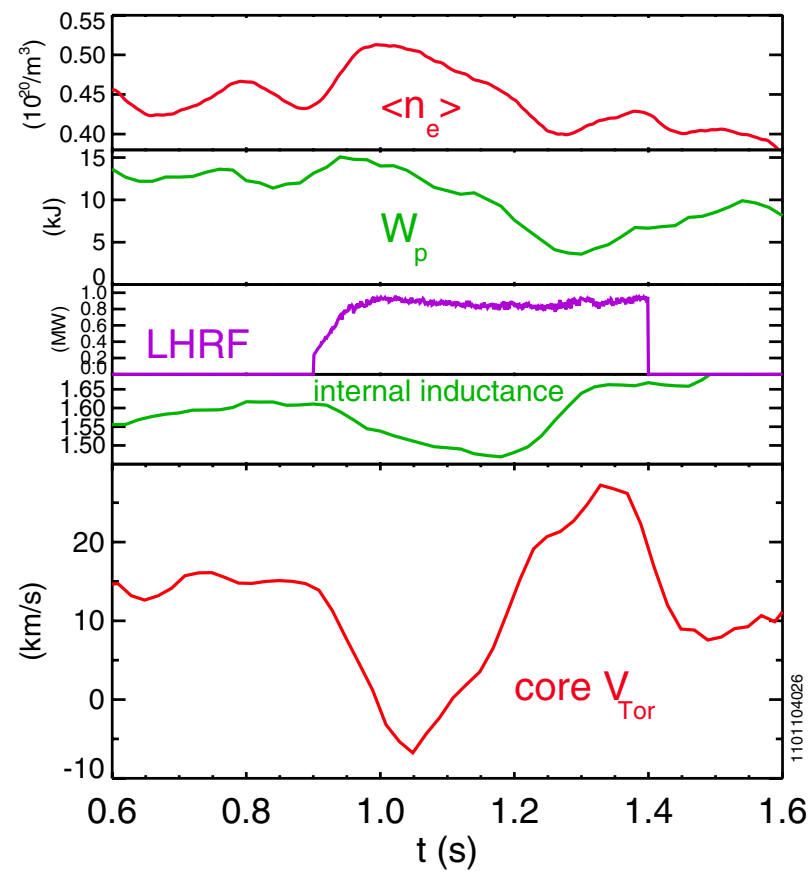

Figure 14. Time histories of the electron density, plasma stored energy, LH power, internal inductance and core rotation velocity for a 5.4 T, 0.55 MA LSN discharge.

evolution is on a much shorter timescale than in figure 1, $\sim 50 \mathrm{~ms}$, close to the energy and momentum confinement times $[56,57]$ and much shorter than the current relaxation time, $\sim 280 \mathrm{~ms}$. This velocity increase associated with application of LH waves (which have greatly reduced core absorption at this high density $[34,60]$ ), develops on a transport time scale, rather than on a current relaxation time scale. The effect on

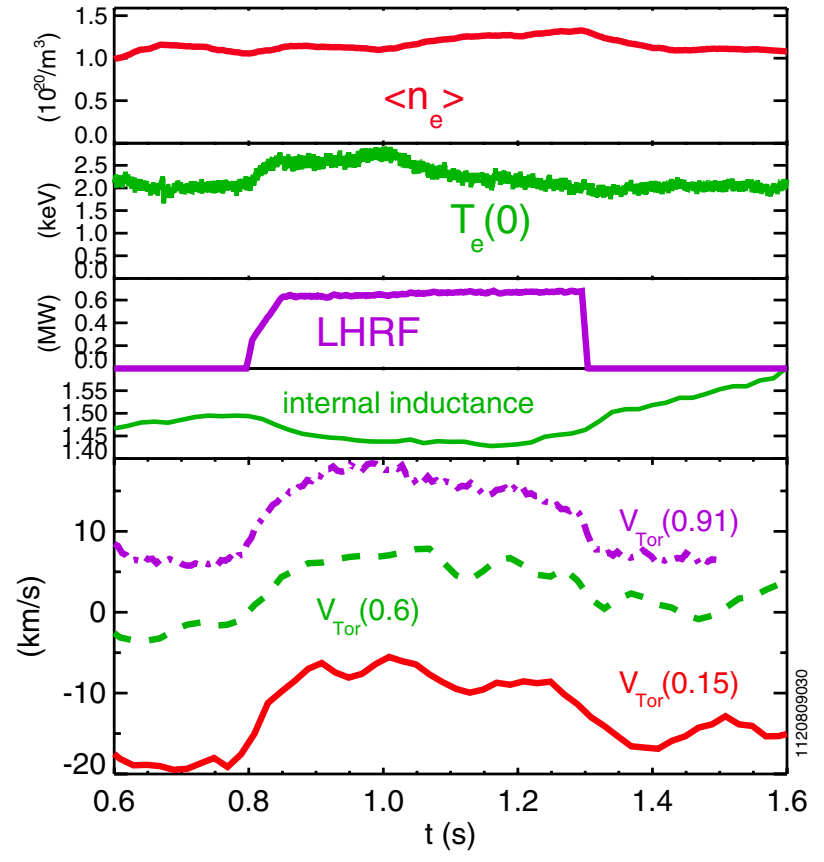

Figure 15. Time histories of the line-averaged electron density, central electron temperature, LH power $\left(n_{\|}=1.9\right)$, internal inductance and rotation velocity in the core (solid), mid-radius (dashed) and edge (dotted-dashed) for a 6.2 T, $0.81 \mathrm{MA}\left(q_{95}=4.9\right)$ USN discharge.

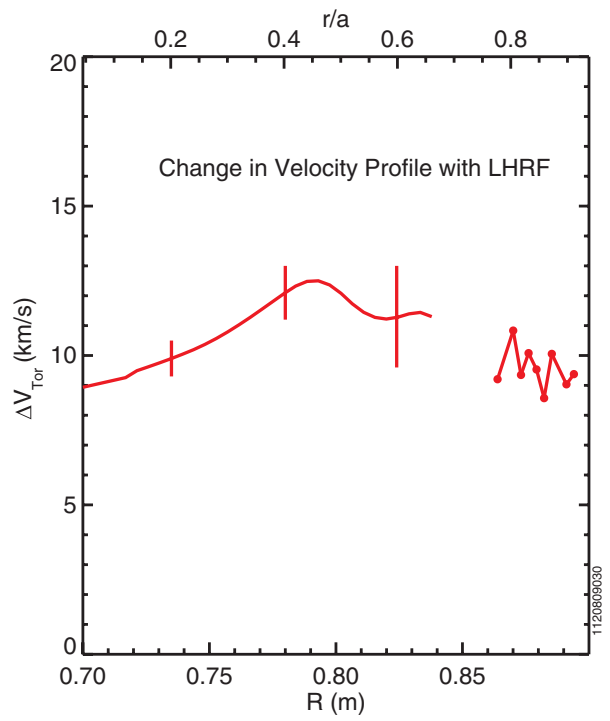

Figure 16. Difference of the radial profiles of the toroidal rotation velocity during and before $\mathrm{LH}$ wave injection for the discharge of figure 15.

the rotation is also seen in the outer regions of the plasma $(r / a \sim 0.9)$, which was not observed in figures 1 and 6 . There was a slight (4\%) drop in the internal inductance during the LHRF injection and a modest increase in the core hard $\mathrm{x}$-ray emission, but very little change in the plasma stored energy or the energy confinement time. The appearance of these LHRF-induced co- rotation L-mode discharges at higher density seems to be independent of $q_{95}$ over the range from 4 to 6 . Shown in figure 16 is the difference of the velocity profiles during and before the LH power pulse. The entire 


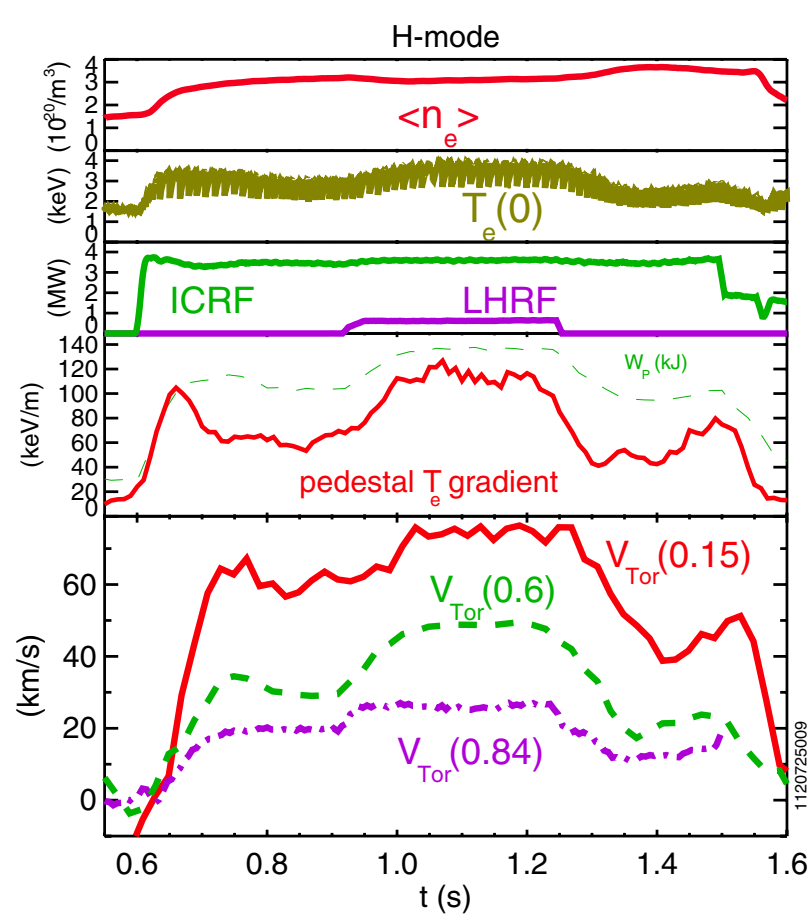

Figure 17. Time histories of the line-averaged electron density, central electron temperature, ICRF and LHRF power, pedestal electron temperature gradient and rotation velocity in the core (solid), mid-radius (dashed) and edge (dashed-dotted) for a 5.4 T, 0.81 MA $\left(q_{95}=4.6\right)$ LSN H-mode discharge.

velocity profile during the LHRF injection is elevated in the co-current direction by about $10 \mathrm{~km} \mathrm{~s}^{-1}$ compared to the ohmic intrinsic rotation profile before the LHRF. This is in contrast to the changes shown in figure 6 where the profiles only varied inside of $r / a=0.5$ with LHRF. A possible scenario for this behaviour is that absorption of the LH waves in the SOL leads to a loss of electrons, which gives rise to a positive edge $E_{\mathrm{r}}$ increment, in this case of about $10 \mathrm{kV} \mathrm{m}^{-1}$, which drives the co-current rotation throughout the plasma.

\section{Velocity changes with LHRF in H-mode target plasmas}

LH waves have also been introduced into H-mode target plasmas, with varying levels of core wave penetration. An example is shown in figure 17 , where $0.65 \mathrm{MW}$ of LHRF power with $n_{\|}=1.9$ were injected into a $0.81 \mathrm{MA}, 5.4 \mathrm{~T}$ $\left(q_{95}=4.6\right) \mathrm{LSN}$ H-mode discharge (accessed with $3.6 \mathrm{MW}$ of ICRF minority heating power), with a line-averaged density of $3.2 \times 10^{20} \mathrm{~m}^{-3}$. The density at the last closed flux surface (LCFS) was $\sim 2 \times 10^{20} \mathrm{~m}^{-3}$, making the LH waves classically inaccessible. Even though at this electron density the LH waves have difficulty accessing past the SOL [60], there was a substantial increase in the central electron temperature and a co-current increment of the toroidal rotation at the time of the LHRF power injection. The rotation evolved on a transport time scale $(\sim 40 \mathrm{~ms})$ and the co-current increase was first seen in the plasma periphery. In fact, a co-current increment in the toroidal rotation at $r / a=0.84$ appeared immediately after the waves were injected. This suggests that the LH waves affect the properties of the edge plasma, and the changes in rotation are due to subsequent transport effects. There were only slight changes in $l_{\mathrm{i}}$ and the loop voltage during the $\mathrm{LH}$ wave injection in this case, and only a very small increase in the core hard $\mathrm{x}$-ray emission.

There was a significant increase in the plasma stored energy and edge pedestal electron temperature gradient (fourth frame) during the LH wave injection. This increase in the pedestal temperature gradient is mainly due to an increase of the electron temperature at the top of the pedestal. An increase in the edge temperature gradient has been shown to give rise to co-current toroidal rotation in the core [55]. There were also substantial increases in the energy confinement time with LHRF, from 29 to $40 \mathrm{~ms}$, and an increase in $\mathrm{H}_{98}$; all of these changes are consistent with an improvement in edge confinement due to the deposition of power in the edge from the LH waves. From a plasma performance perspective, the additional LH power is quite efficient; for an $18 \%$ increase in auxiliary power there was a $28 \%$ increase in the plasma stored energy (fourth frame), a $38 \%$ increase in the global energy confinement time and a $32 \%$ increase in $\mathrm{H}_{98}$, from 0.69 to 0.92 . The cause of such a relatively large effect from such a small amount of additional power is not clear. Indeed LHRF power injection into these high density H-modes does not always show such large confinement improvements, and in some discharges the confinement improvement was lost while the LHRF power was still on. Clues for this behaviour may be found from examination of effects observed in the edge region.

In high density H-mode discharges with LHRF power injection, significant changes are observed in the lab frame central frequency of the quasi-coherent mode (QCM). This mode exists only in the pedestal/near SOL region on the low-field side (LFS), and is responsible for the reduced confinement of particles and impurities in EDA H-mode as compared to ELM-free H-modes [61]. In the lab frame, this mode propagates in the electron diamagnetic drift (EDD) direction with $k_{\theta} \sim 1.5-2 \mathrm{~cm}^{-1}$ (at the LFS mid-plane). Associated with the increase in stored energy obtained with LHRF power injection, the central frequency of this mode is seen to downshift from $\sim 100 \mathrm{kHz}$ to roughly half that frequency, as seen in figure 18. (This is from a discharge similar to that shown in figure 17.) Assuming that this is the result of a Doppler shift only $\left(\mathrm{k}_{\theta}\right.$ changes by $\left.<10 \%\right)$, the change in QCM frequency is consistent with (1) an incremental co-current increase in toroidal rotation at the QCM radial location of $\Delta V_{\phi}=\Delta\left(\omega_{0}^{\mathrm{QCM}} / k_{\theta}^{\mathrm{QCM}}\right)\left(B_{\phi} / B_{\theta}\right) \sim 9 \mathrm{~km} \mathrm{~s}^{-1}$, (2) an increase of $\sim 7 \mathrm{kV} \mathrm{m}^{-1}$ in the presumed negative $E_{r}$ at the QCM location, leading to a $\sim 1.7 \mathrm{~km} \mathrm{~s}^{-1}$ decrease in $V_{E \times B}$ or (3) some combination of these. Since a $\sim 5-$ $7 \mathrm{~km} \mathrm{~s}^{-1}$ incremental co-current change in $V_{\phi}$ is measured spectroscopically in this region (see figure 17), most of the change in the QCM frequency is likely due to this co-current velocity change. Similar discharges that have little or no confinement improvement with LHRF power injection show no such frequency downshift; when the confinement increment is lost while the power is still on, the QCM central frequency up-shifts to the larger frequency on a $10-20 \mathrm{~ms}$ timescale. These results suggest the possibility of using LHRF as a tool to affect plasma rotation and confinement via direct modification of quantities at the plasma edge. Note that many prior C-Mod $\mathrm{H}$-modes with LHRF have shown confinement improvements 


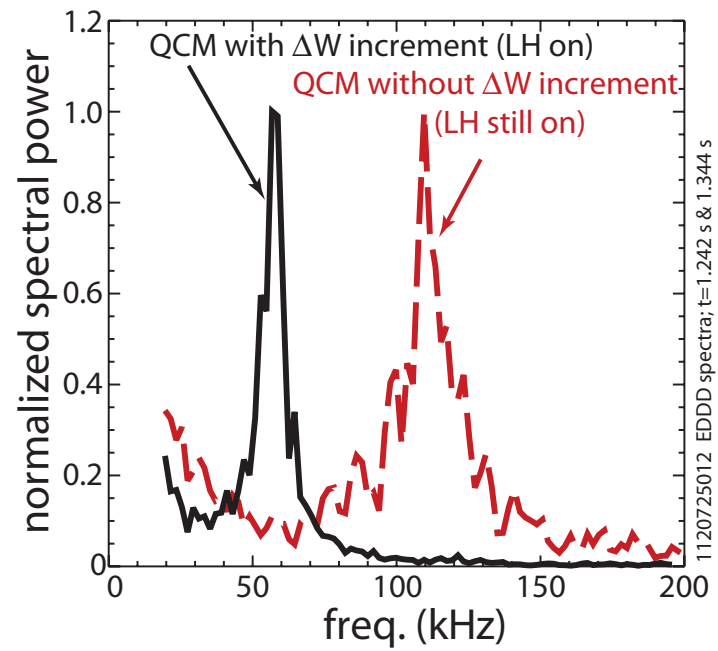

Figure 18. Lab frame frequency changes seen in the QCM, as measured using gas puff imaging. In black is the normalized fluctuation power spectrum during a $5 \mathrm{~ms}$ time interval with $0.6 \mathrm{MW}$ of LHRF power injected and a 25-30\% increase in stored energy brought about by the LHRF wave injection. The red dashed curve is the spectrum while the LHRF power was still on, but the incremental increase in the stored energy had been lost. The measurements were made just below the outboard midplane and map to a location $\sim 8 \mathrm{~mm}$ inside the LCFS.

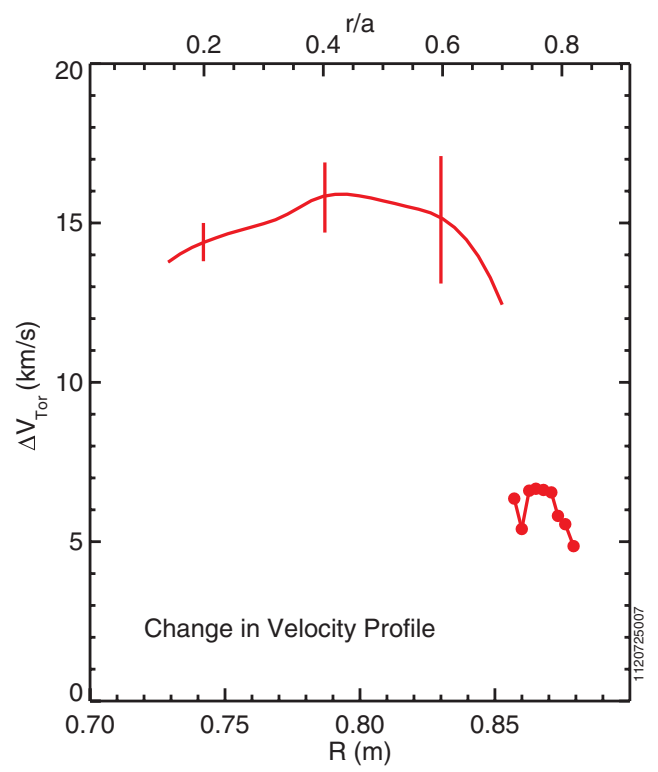

Figure 19. The change of the toroidal rotation velocity profile for a discharge similar to that shown in figure 17, the difference between the ICRF + LHRF interval and the ICRF only portion.

in the absence of a co-current change in edge toroidal rotation [23]. Reasons for these differences are not clear, although there may be significant differences in the quality of LH wave absorption.

The increment in the velocity profile, observed after the addition of steady LHRF power to an ICRF only H-mode discharge, is shown in figure 19. (This is from a discharge similar to that shown in figures 17 and 18.) For ICRF Hmode plasmas, the toroidal rotation is strongly co-current over the entire core region [57]. Addition of LHRF power to this

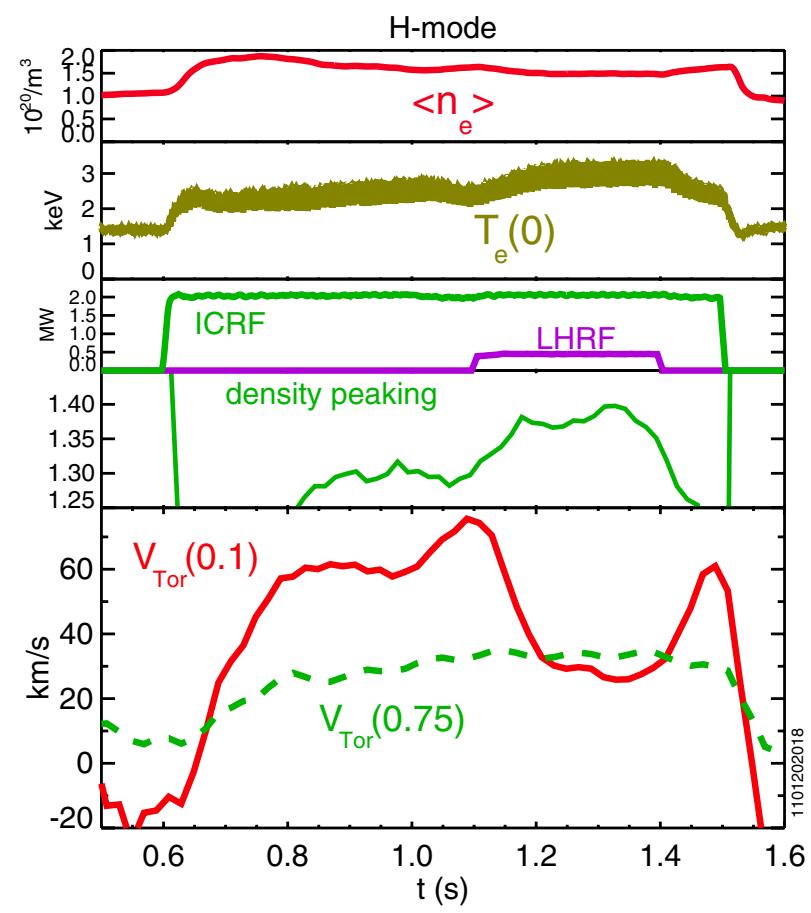

Figure 20. Time histories of the line-averaged electron density, central electron temperature, ICRF and LHRF power, electron density peaking (ratio of core to edge) and rotation velocity in the core (solid) and edge (dashed) for a 5.4 T, 0.62 MA $\left(q_{95}=6.3\right)$ USN $\mathrm{H}$-mode discharge.

plasma further increases the core region by about $15 \mathrm{~km} \mathrm{~s}^{-1}$ in the co-current direction. The velocity change in the edge region was about a factor of two lower. The likely explanation for this co-current rotation drive is similar to the standard $\mathrm{H}$ and I-mode picture. The additional power gives rise to an enhanced $E_{\mathrm{r}}$ shear, which reduces turbulence leading to an improvement in confinement and a steepening of the edge temperature gradient in the pedestal, which then drives the toroidal rotation via the residual stress [55].

Instances of counter-current rotation increments due to LH waves injected into $\mathrm{H}$-mode plasmas have also been observed [16,23]. These cases may have some degree of wave accessibility inside the LCFS. One such example is shown in figure 20, which demonstrates the effects of $0.45 \mathrm{MW}$ of LHRF power with $n_{\|}=1.9$ injected into a $0.62 \mathrm{MA}, 5.4 \mathrm{~T}\left(q_{95}=6.3\right)$ USN H-mode discharge at a line-averaged electron density of $1.6 \times 10^{20} \mathrm{~m}^{-3}\left(1.4 \times 10^{20} \mathrm{~m}^{-3}\right.$ at the LCFS $)$, accessed with $2.0 \mathrm{MW}$ of ICRF minority heating power. There is a distinct counter-current increment in the core rotation, which evolves on a time scale of $\sim 50 \mathrm{~ms}$, similar to the global energy confinement time for this discharge, $\sim 34 \mathrm{~ms}$. In contrast to the previous case (figures 17-19), there was no change in rotation at $r / a=0.8$. For this plasma there was no change in the internal inductance, core hard x-ray emission or the loop voltage due to the LH wave injection. The decrease in the core rotation velocity is correlated with a peaking of the electron density profile. While there was an increase in the core electron temperature, there was no peaking of the temperature profile, and no change in the stored energy or energy confinement time. This peaking of the density profile (fourth frame of figure 20) is the result of an edge density pedestal reduction [23]. A 


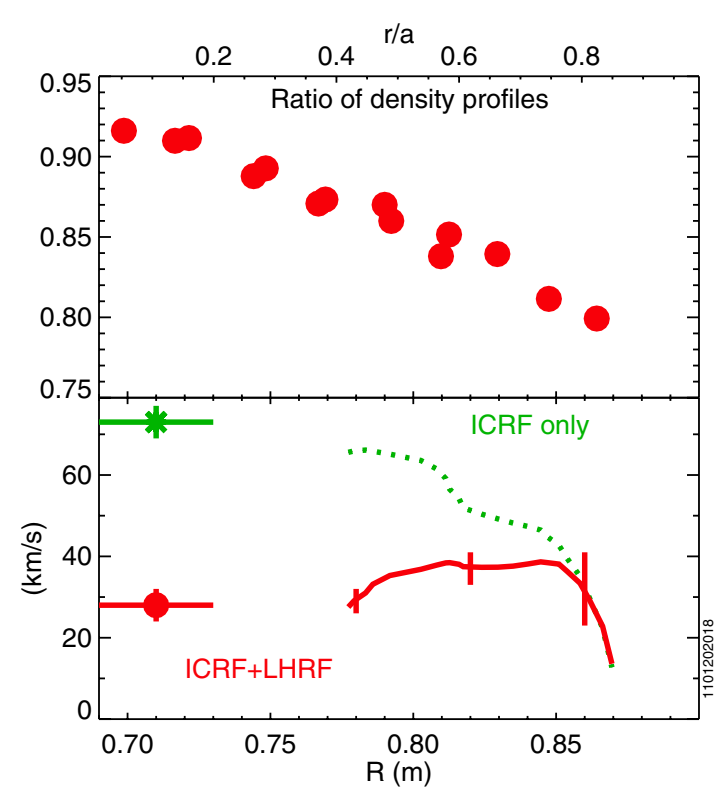

Figure 21. Ratio of the electron density profile with LH waves to the profile with ICRF only (top) for the discharge of figure 20. In the bottom frame are the toroidal rotation profiles, just before (1.05-1.1 s, asterisk and dotted line) and during (1.2-1.4 s, dot and solid line) LHRF.

comparison between electron density and toroidal rotation profiles during the ICRF $\mathrm{H}$-mode phase, and with additional LHRF power, is shown in figure 21. In the top frame is shown the ratio of the electron density profile during the time interval with LHRF to the profile with ICRF only, and in the bottom frame are the rotation velocity profiles during the ICRF only phase (1.05-1.1 s) and the additional LHRF portion (1.2-1.4 s). The electron density profile clearly becomes more peaked with the LHRF, while the velocity profile flattens. During the ICRFonly portion of the discharge, the toroidal rotation velocity spatial profile is centrally peaked, which suggests the presence of an inward momentum pinch [56,57]. With the addition of the LHRF power, the rotation profile flattens, which is consistent with a suppression of the inward momentum pinch. However, it is not possible to distinguish between a reduction in the momentum pinch and a reduction of the intrinsic torque in the core.

Not all discharges with these $\mathrm{H}$-mode target conditions exhibit the rotation drop or density profile peaking. Shown in figure 22 is a comparison of two similar ICRF H-mode discharges with additional LHRF power injection (0.93 MW with $\left.n_{\|}=2.3\right)$. Both 0.61 MA, 5.4 T $\left(q_{95}=6.6\right) \mathrm{USN}$ plasmas had a target density of $1.8 \times 10^{20} \mathrm{~m}^{-3}$ during the $\mathrm{H}$-mode phase. The discharge which experienced a decrease in the central rotation with LHRF power showed an increase in the electron temperature and a peaking of the electron density profile. It's not clear what led to this effect in one plasma and not the other, but there is a correlation between a flattening of the velocity profile and a peaking of the electron density profile. One possible difference is that the discharge without the density peaking and rotation drop had a substantially higher radiated power (by about 25\%) at the time of the LHRF pulse, perhaps because this plasma was later in the day, after the boronization wall conditioning had started to wear off.

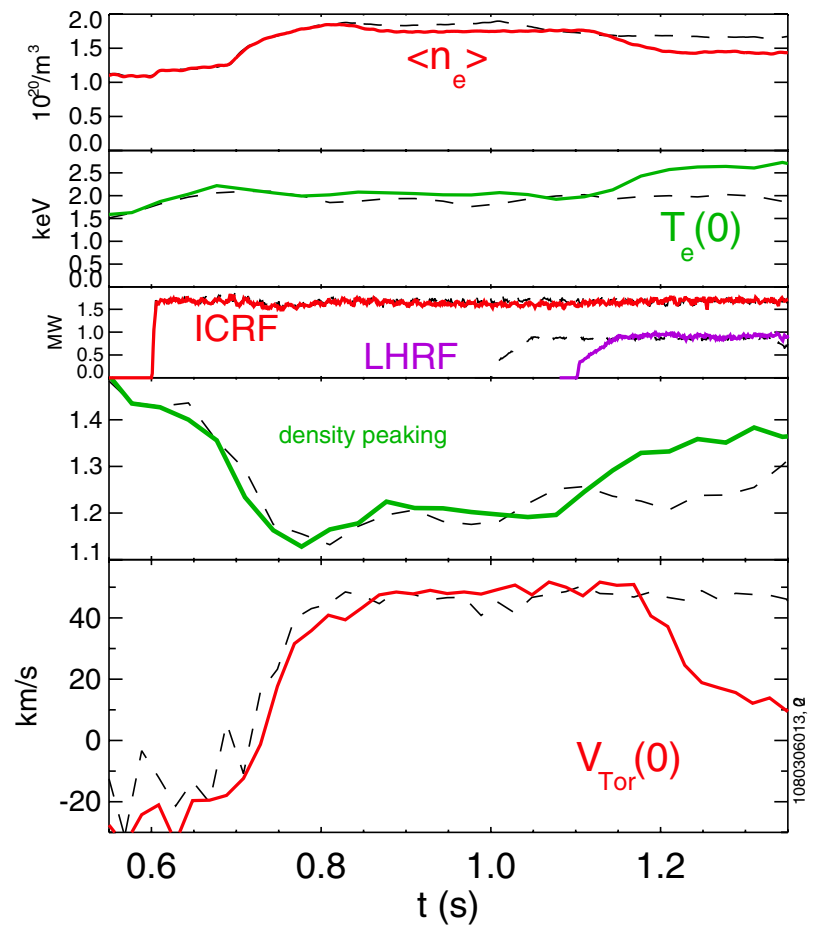

Figure 22. Parameter time history comparison of two $0.61 \mathrm{MA}$, 5.4 T USN discharges with additional LHRF power. From top to bottom, the line-averaged electron density, central electron temperature, ICRF and LHRF power, electron density peaking (ratio of core to edge) and rotation velocity in the core.

It has been shown [23] that excess core radiation prevents LH-induced modification of the pedestal. The correlation between the counter-current increment in the core rotation (indicative of a flattening of the velocity profile) and the peaking of the electron density profile is demonstrated in figure 23 . The change in the core rotation velocity with LHRF power is shown as a function of the electron density peaking factor, here defined as the ratio between the central density and that at the $95 \%$ flux surface. There is a strong anti-correlation between the two profile shapes.

For the sake of completeness, two groups of points from $\mathrm{H}$-mode plasmas similar to those shown in figures 17-23 have been added to the operational space plot in the $n_{\mathrm{e}}-q_{95}$ plane, and are shown in figure 24 . There is a rich variety of LHRFinduced rotation behaviour over the $\mathrm{C}$-Mod operating range.

\section{Discussion}

The parameters and qualitative changes from the variety of discharges into which LH waves were injected, catalogued in figure 24, are summarized in table 2. This compares the relevant time scales, profile shapes, characteristic changes, parameters ranges and rotation drive mechanisms for LHRF plasmas. At the top is given the figure number of a plasma which exemplifies a particular type of response to LHRF injection. The first three rows give the relevant time scales for the core velocity change, current relaxation and energy confinement, respectively. The next three rows indicate the change/increment direction of the rotation velocity and profile. The following rows list other relevant parameter changes and plasma conditions. 


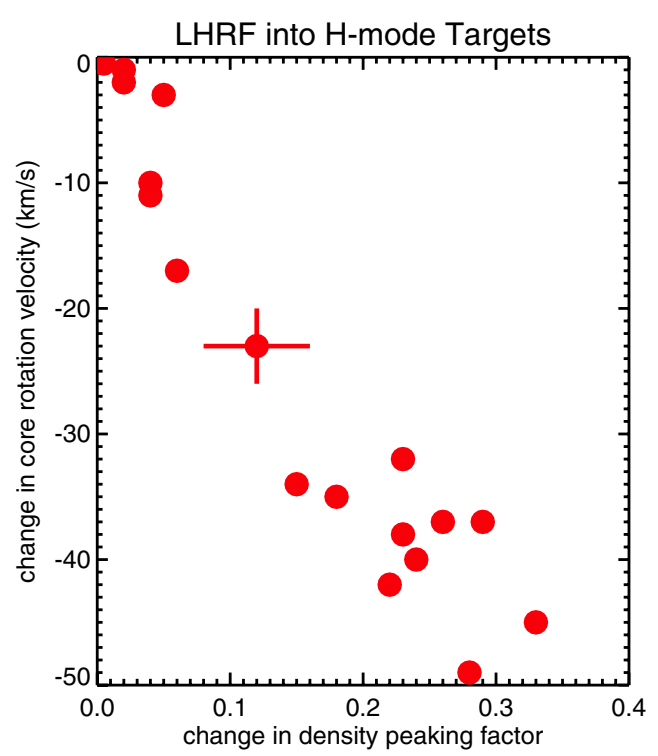

Figure 23. The change in the core rotation velocity as a function of the change in the electron density peaking factor for $\mathrm{H}$-mode discharges with LHRF.

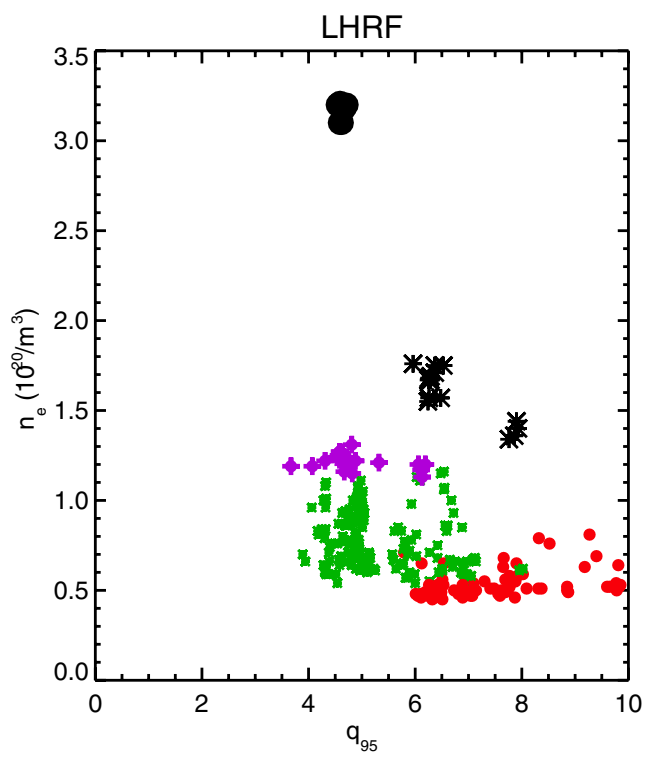

Figure 24. The plot of figure 10 expanded to include $\mathrm{H}$-mode target plasmas with LHRF. Dots indicate a velocity increment in the co-current direction while asterisks are for a counter-current change.

There appear to be two general classes of LHRFinduced toroidal rotation changes: those with good core absorption of the LH waves with concomitant current drive and those cases where the LH wave energy is deposited in the periphery. In low density $\left(<1.1 \times 10^{20} \mathrm{~m}^{-3}\right) \mathrm{L}-$ mode target plasmas with good LH wave accessibility and absorption (red and green points in figures 9, 10 and 24, columns 1 and 2 in table 2), the core rotation (only inside of $r / a \sim 0.5$ ) evolves on a current relaxation time scale (hundreds of ms, see row 2). These discharges also have significant changes in the internal inductance and hard x-ray emission. For the lowest density, low current discharges in this class, the rotation change is in the co-current direction, while for higher density, high current plasmas, the rotation increment is counter-current. This may be due to an electron pinch (a direct LH wave effect, more effective with more trapped electrons) or through changes in the current density profile, which can change the sign of the residual stress, and hence the rotation direction (discussed below). The counter- increment plasmas (column 1 in table 2) had sawtooth oscillations, while in the co-increment discharges (column 2), the sawteeth were suppressed due to a significant change in the $q$ profile. Table 3 summarizes the sawtooth behaviour for a database of 243 low density target plasmas with LHCD. No co-increment discharges exhibited sawtooth oscillations while all sawtoothing discharges underwent counter-current rotation changes. Only a few counter-increment plasmas were without sawteeth.

In high density target plasmas $\left(n_{\mathrm{e}}>1.2 \times 10^{20} \mathrm{~m}^{-3}\right)$, where the LH waves are thought to be damped in the plasma periphery, the rotation profile evolves on a transport time scale (tens of ms, row 3, table 2). The influence of the LH waves is indirect, causing changes in the SOL and/or edge plasma, which affect the core plasma, on a transport time scale. (See table 2 columns 3-7)

Consider first the behaviour shown in figures 15 and 16 (third column table 2), which is arguably the least complex of all of these cases (purple points in figures 9, 10 and 24). The LH waves, which have poor core absorption at this density [62], are damped in the plasma edge. An increase in the SOL pressure has been observed on edge probes, indicating an edge perturbation. At the same time, there was an increase of the radial electric field, $E_{\mathrm{r}}$, at $r / a=0.95$, from $+20 \mathrm{kV} \mathrm{m}^{-1}$ to $+30 \mathrm{kV} \mathrm{m}^{-1}$, associated with changes in the toroidal rotation velocity (see figures 15 and 16). This increase is consistent in sign with edge electron orbit losses. The co-current toroidal velocity evolves on an L-mode momentum transport time scale $[56,57]$, a few tens of ms, similar to the energy confinement time.

The results of figures 17-19 (fourth column table 2, large black dots in figure 24) are consistent in magnitude and direction with earlier observations of toroidal rotation in ICRFheated $\mathrm{H}$ - and I-mode plasmas [55], where the co-current change in rotation has been associated with an increase in the pedestal temperature gradient. A co-current perturbation is first seen at the plasma periphery, and then propagates into the core. This is accompanied by increases in the plasma stored energy and $\mathrm{H}$ factor. The important issue is the mechanism by which injection of LH waves (which do not routinely penetrate into the plasma at these high densities) leads to an increase of the pedestal temperature gradient. The underlying concept for the last two cases is that changes to the momentum flux through $\Pi_{r}$, due the LH waves damped in the plasma periphery, lead to an intrinsic torque (final row table 2).

For figures 20-23 (table 2 columns 5-7, large black asterisks in figure 24), there is a flattening of the rotation profile which evolves along with a peaking of the electron density profile, suggesting a reduction of inward momentum transport, either due to a reduction of an inward momentum pinch, or to a modification of $\Pi_{r}$. This is in qualitative agreement with the predicted trends of the inward turbulent equipartition [63] and Coriolis [64] momentum pinches, which scale as $R / L_{n}$. Scaling of the momentum pinch with $R / L_{n}$ is commonly observed [39,65-70]. For the discharge of figure $20, R / L_{n}$ at 
Table 2. A summary of qualitative changes and parameters for LHRF discharges. 'n' stands for no and 'y' represents yes.

\begin{tabular}{|c|c|c|c|c|c|c|c|}
\hline Example & Figure 1 & Figure 2 & Figure 15 & Figure 17 & Figure 20 & Figure 22 & Figure 22 \\
\hline$\tau_{\mathrm{V}_{\phi}}(\mathrm{s})$ & 0.2 & $\sim 0.2$ & 0.05 & 0.04 & 0.05 & 0.05 & 一 \\
\hline$\tau_{\mathrm{CR}}(\mathrm{s})$ & 0.15 & 0.10 & 0.28 & 0.55 & 0.25 & 0.26 & 0.24 \\
\hline$\tau_{\mathrm{E}}(\mathrm{s})$ & 0.03 & 0.03 & 0.04 & 0.04 & 0.03 & 0.03 & 0.03 \\
\hline$\Delta V_{\text {core }}$ & cntr & $\mathrm{co}$ & $\mathrm{co}$ & $\mathrm{co}$ & cntr & cntr & $\mathrm{n}$ \\
\hline$\Delta V$ profile & core & core & entire & entire & core & core & $\mathrm{n}$ \\
\hline$\Delta V_{\text {edge }}$ & $\mathrm{n}$ & $\mathrm{n}$ & co & co & $\mathrm{n}$ & $\mathrm{n}$ & $\mathrm{n}$ \\
\hline$\Delta \nabla T_{\text {ped }}$ & $\mathrm{n}$ & $\mathrm{n}$ & $\mathrm{n}$ & $\mathrm{y}$ & $\mathrm{n}$ & $\mathrm{n}$ & $\mathrm{n}$ \\
\hline$\Delta \nabla n_{\text {core }}$ & $\mathrm{n}$ & $\mathrm{n}$ & $\mathrm{n}$ & $\mathrm{n}$ & $\mathrm{y}$ & $\mathrm{y}$ & $\mathrm{n}$ \\
\hline Target $q_{95}$ & 3.7 & 7.7 & 4.9 & 4.7 & 6.3 & 6.5 & 6.4 \\
\hline Target $n_{\mathrm{e}}\left(10^{20} \mathrm{~m}^{-3}\right)$ & 0.7 & 0.7 & 1.2 & 3.3 & 1.6 & 1.8 & 1.9 \\
\hline Target regime & L- & L- & L- & $\mathrm{H}-$ & $\mathrm{H}-$ & $\mathrm{H}-$ & H- \\
\hline Core wave abs & $\mathrm{y}$ & $\mathrm{y}$ & low & $\mathrm{n}$ & $\mathrm{n}$ & $\mathrm{n}$ & $\mathrm{n}$ \\
\hline$\Delta q(r)$ & low & $\mathrm{y}$ & $?$ & $\mathrm{n}$ & $\mathrm{n}$ & $\mathrm{n}$ & $\mathrm{n}$ \\
\hline$q_{0}$ & 0.7 & 1.8 & $<1$ & $<1$ & $<1$ & $<1$ & $<1$ \\
\hline$\Delta l_{\mathrm{i}}$ & $\mathrm{y}$ & $\mathrm{y}$ & low & $\mathrm{n}$ & $\mathrm{n}$ & $\mathrm{n}$ & $\mathrm{n}$ \\
\hline Sawteeth & $\mathrm{y}$ & $\mathrm{n}$ & $\mathrm{y}$ & $\mathrm{y}$ & $\mathrm{y}$ & $\mathrm{y}$ & $\mathrm{y}$ \\
\hline Hard x-ray & $\mathrm{y}$ & $\mathrm{y}$ & $\mathrm{y}$ & $\mathrm{n}$ & $\mathrm{n}$ & $\mathrm{n}$ & $\mathrm{n}$ \\
\hline Drive & $e_{\text {pinch }} ?$ & $\nabla q$ & $V_{\text {edge }} ?$ & $\nabla T_{\text {ped }}$ & $\nabla n_{\text {core }}$ & $\nabla n_{\text {core }}$ & - \\
\hline Momentum flux & source? & $\Pi_{r}$ & $\Pi_{r}$ & $\Pi_{r}$ & $V_{\text {pinch }} ?$ & $V_{\text {pinch }} ?$ & - \\
\hline
\end{tabular}

Table 3. Number of discharges, showing correlation between sawteeth and rotation increment direction with LHCD for low density L-mode target plasmas.

\begin{tabular}{lcl}
\hline & Sawteeth & No sawteeth \\
\hline co- & 0 & 89 \\
counter- & 146 & 8 \\
\hline
\end{tabular}

the mid-radius increased from 2.4 to 3.1 during the $\mathrm{LH}$ wave injection, while the velocity profile flattened due to a reduction of the momentum pinch. The peaking of the electron density profile is actually due to a reduction of the pedestal density, with very little change in the central density [23]. The LH waves, which are absorbed in the plasma periphery at these high densities, affect the edge density pedestal, likely through a change in the radial particle transport. In contrast to the two previous cases, changes to the momentum flux could be due to changes in the momentum pinch term (final row table 2).

The examples with good core LHCD power deposition shown in sections 2 and 3 (table 2 columns 1 and 2) will be discussed next, with the goal of explaining the bi-directional rotation changes. A comparison will first be made of discharges at the extremes of the plasma current scan shown in figure 4. The LHCD wave deposition profiles for two such plasmas (from section 2), with currents of $0.32\left(q_{95}=9.6\right)$ and 0.91 MA $\left(q_{95}=3.7\right)$, and with $\left\langle n_{\mathrm{e}}\right\rangle=0.7 \times 10^{20} \mathrm{~m}^{-3}$, have been calculated from GENRAY/CQL3D [71,72], and are shown in figure 25. LHCD power levels for the two cases were 0.72 and $0.9 \mathrm{MW}$, respectively. For the $0.32 \mathrm{MA}$ case, the waves are damped closer to the plasma core, and the driven current is estimated to be $0.23 \mathrm{MA}$, over $2 / 3$ of the total current. For the $0.91 \mathrm{MA}$ discharge, the total driven current is calculated to be $0.5 \mathrm{MA}$.

Shown in figure 26 are the electron density and temperature profiles for these same two plasmas, during the LH wave injection. The electron density profiles are nearly identical, while in the high current case, the temperature is considerably higher, and the profile is broader. For both plasmas, $Z_{\text {eff }} \sim 4$. Values for the collisionality, $v_{*}$, at the

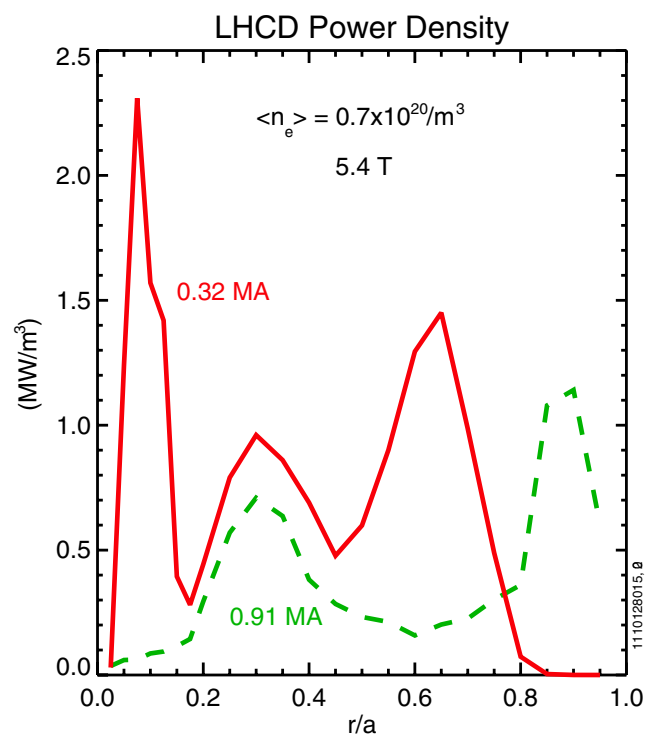

Figure 25. Calculated LHCD power deposition profiles for $0.32 \mathrm{MA}$ (red solid) and 0.91 MA (green dashed) discharges.

mid-radius are 0.2 (0.91 MA) and $1.3(0.32 \mathrm{MA})$, on either side of the linear ohmic confinement (LOC)/saturated ohmic confinement (SOC) boundary, determined to be $v_{*} \sim 0.35$ $[42,48]$. These two target plasmas are in collisionality regimes dominated by different turbulence types: TEMs for low $v_{*}$ and ITG modes for high $v_{*}$.

Current density profiles have been determined from EFIT calculations constrained by MSE observations [27, 28] enabled by a diagnostic neutral beam [25]. The inverse rotational transform $q$, magnetic shear $(\hat{s}=r / q \partial q / \partial r)$ and magnetic shear scale length $\left(L_{s} \equiv R_{0} q / \hat{s}\right)$ profiles, for the same two discharges, plus an intermediate current case (0.58 MA), are shown in figure 27. For the 0.91 MA plasma (which was sawtoothing throughout the LHCD pulse), the $q$ profile (top frame) increases monotonically, with $q_{0}$ well below 1 at the magnetic axis. The zero crossing at $0.75 \mathrm{~m}$ is consistent with the measured sawtooth inversion radius. For the 0.32 MA case, 


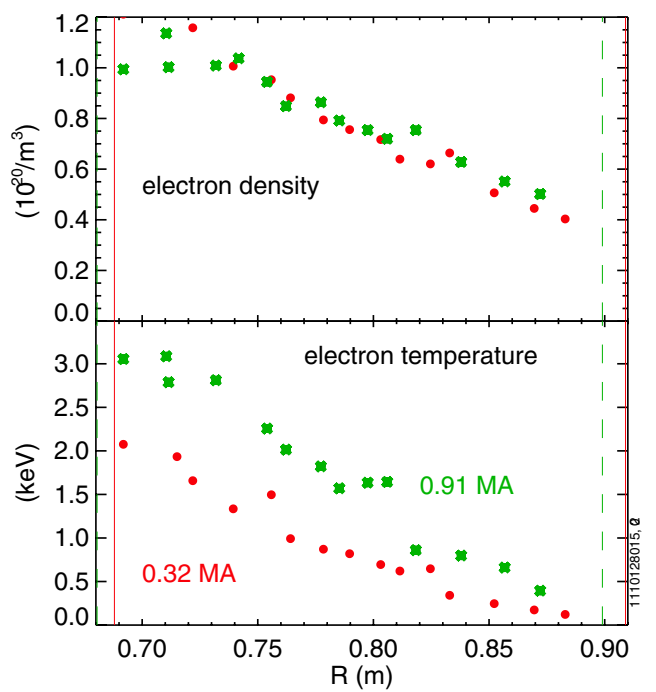

Figure 26. The electron density (top) and temperature (bottom) for $0.91 \mathrm{MA}$ (green asterisks) and 0.32 MA (red dots) discharges with LHCD. Locations of the magnetic axis and LCFS are shown as vertical lines, solid red for the $0.32 \mathrm{MA}$ case and green dashed for $0.91 \mathrm{MA}$.

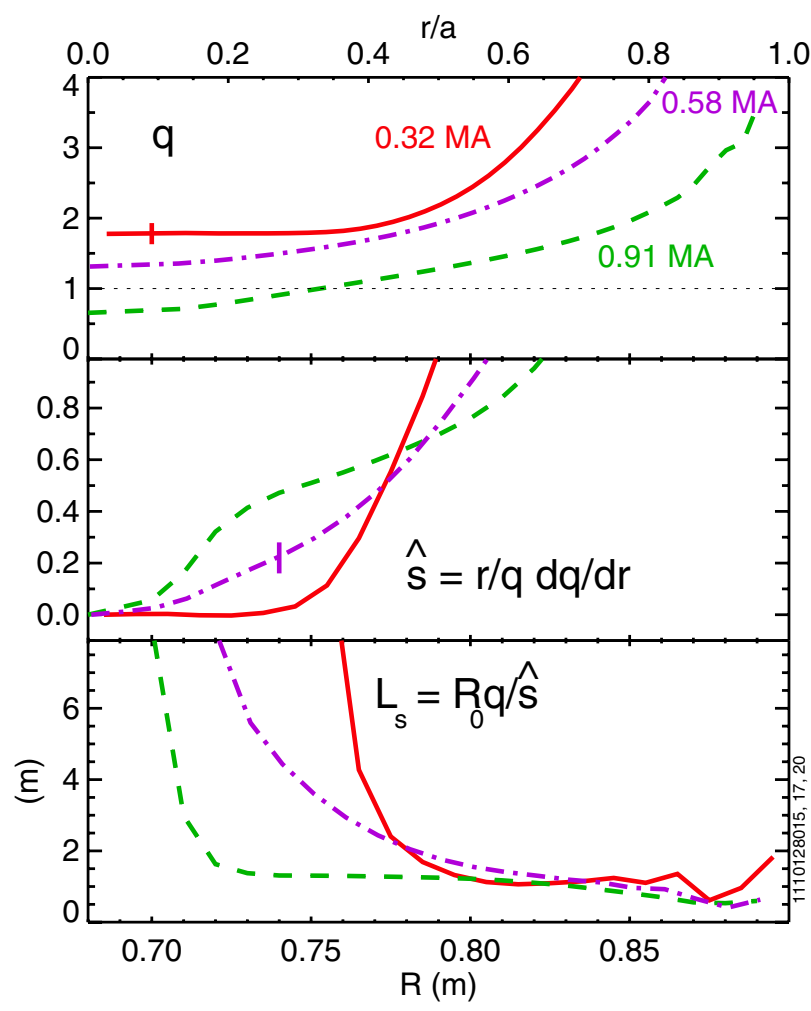

Figure 27. From top to bottom, the inverse rotational transform $q$, magnetic shear and $L_{s}$ profiles for $0.32 \mathrm{MA}$ (red solid), $0.58 \mathrm{MA}$ (purple dashed-dotted) and 0.91 MA (green dashed) discharges with LHCD. Typical error bars are shown.

the $q$ profile is flat in the core, with a value just under 2 ; for the 0.58 MA example, $q_{0} \sim 1.3$. This is consistent with the lack of sawtooth oscillations for these latter two discharges. Another difference between these cases is the magnetic shear (middle frame); for the 0.91 MA plasma, $\hat{s}$ in the core $(R=0.75 \mathrm{~m}$, $r / a \sim 0.3$ ) was about 0.5 , for the $0.58 \mathrm{MA}$ case the core $\hat{s}$

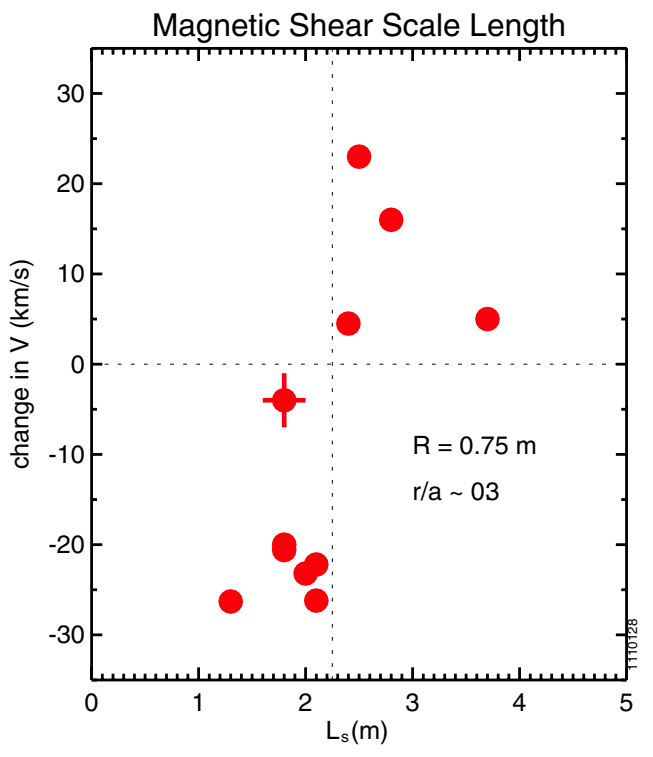

Figure 28. The change in the core rotation velocity with LHCD as a function of the magnetic shear scale length at $r / a \sim 0.3$.

was $\sim 0.2$, while for the $0.32 \mathrm{MA}$ discharge, $\hat{s}$ was near 0 . Interestingly, all three $\hat{s}$ profiles converge near the mid-radius, which is close to the rotation profile stagnation point shown in figure 6 . For the low current case, $\hat{s}$ hovers $\sim 0$ which gives rise to an exploding $L_{s}$ (bottom frame) inside $R=0.75 \mathrm{~m}$ $(r / a \sim 0.3)$. For the $0.91 \mathrm{MA}$ plasma, the value of $L_{s}$ at $r / a \sim 0.3$ was $\sim 1.5 \mathrm{~m}$, and $\sim 3.5 \mathrm{~m}$ for the 0.58 MA discharge.

The connection between the magnetic shear scale length and direction of rotation increment is illustrated in figure 28 where the change in rotation velocity with LHCD is shown as a function of the average value of $L_{s}$ near $r / a \sim 0.3$. There is an abrupt change in the LHCD rotation increment, going from counter- to co-, near $L_{s} \sim 2.3 \mathrm{~m}$, exhibiting a sort of threshold behaviour. The co-current rotation change point from the 0.32 MA discharge had $L_{s} \sim 20 \mathrm{~m}$ and is off scale. Typical error bars are shown. It should be noted that both USN and LSN points are intermixed, in contrast to the results shown in figure 4.

A similar threshold is seen as a function of the central inverse rotational transform, as is illustrated in figure 29, where the change in core rotation frequency is shown depending on $q_{0}$. The points occupy two quadrants: counter-current rotation changes with $q_{0}<1$ and co-current changes with $q_{0}>1$. The null in $\Delta \omega$ is close to $q_{0} \sim 1$, consistent with the results of table 3 . The use of the rotation frequency allows for direct comparison with the results from other devices; also shown in figure 29 is a point from EAST [17], which is in qualitative agreement with the C-Mod points.

These changes in the $q$ profiles are significant, because the structure of the residual stress, $\Pi_{r}$ [52-54] (which gives rise to an intrinsic torque via $\nabla \cdot \Pi_{r}$ ), and the turbulent acceleration [73], both depend sensitively on the magnetic shear, $\hat{s}$, and mode structure via ion-acoustic coupling. In particular, both drivers of intrinsic rotation require symmetry breaking, so as to set a finite value of $\left\langle k_{\perp} k_{\|}\left|\hat{\Phi}_{k}\right|^{2}\right\rangle$, necessary for intrinsic torque. Here the bracket refers to a spectral average. To this end, the intrinsic torque can change noticeably [74] as 


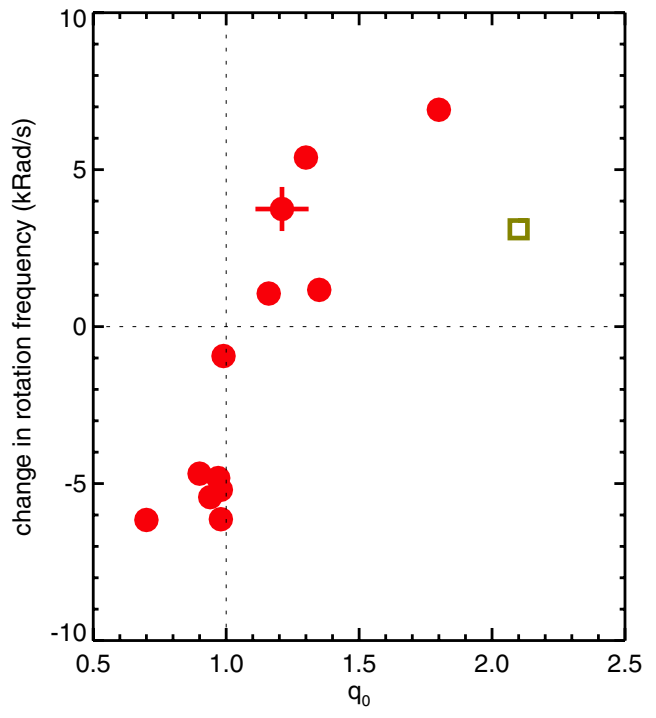

Figure 29. The change in the core rotation frequency with LHCD as a function of $q_{0}$. Dots: C-Mod, box: EAST.

$q^{\prime}$ and $\hat{s}$ drop, and $q^{\prime \prime}$ increases. Furthermore, the intrinsic torque for the normal magnetic shear and 'flat $q$ ' cases can differ significantly, although the case may be understated. For weak shear (flat $q$ profiles), non-resonant modes become increasingly important. Recent work [75] has characterized the structure of non-resonant modes and noted that they are best thought of as extended convective cells, with little, if any, similarity to the familiar resonant modes. Further studies [76] have revealed that these non-resonant modes make a substantial contribution to the turbulent heat flux. Thus, there is every reason to expect that the non-resonant modes play an important role in the non-diffusive radial flux of toroidal momentum and the associated Reynolds stress $\left\langle\tilde{v}_{r} \tilde{v}_{\phi}\right\rangle$. Studies of the effect of non-resonant modes on momentum transport are ongoing and will be discussed in future publications. For now, it should be emphasized that the change in intrinsic torque as LHCD induced current increases and $q(r)$ flattens is quite likely related to the contributions of non-resonant modes. Previous work on turbulence driven intrinsic torque has ignored the effect of non-resonant modes.

In order to explore the dependence of LHCD rotation on turbulence, linear gyrokinetic simulations were performed using the gs 2 code [77]. These calculations were performed for the 0.71 and $0.42 \mathrm{MA}$ discharges shown in figure 6, with well characterized rotation increments in the counter- and cocurrent directions, respectively. Measured electron density and temperature, ion temperature, rotation velocity and $q$ profiles were available for both cases. Linear instabilities were restricted to $k_{\theta} \rho_{\mathrm{i}} \leqslant 0.5$, where it was observed that there were common local maxima of the instability spectra. gs2 assumes a Maxwellian electron distribution function, which is not accurate in plasmas with significant LHCD. An estimation of the effect of the non-Maxwellian tail was performed using a high width, low intensity second electron distribution, and it was found that this had a negligible effect on the most unstable linear modes. It was seen that the 0.42 MA discharge had a small section of turbulence propagating in the ion diamagnetic direction, whereas instability propagation in the $0.71 \mathrm{MA}$ case was completely in the electron diamagnetic direction. Overall the mode structure differences between the two cases were not obviously significant. The most unstable mode at $r / a \sim 0.5$ was selected and sensitivity studies were performed on the most common turbulence driving terms. The two parameters where there were marked differences in the spectra for the two current cases were $a / L_{n}$ and $a / L_{T_{\mathrm{i}}}$. For the $0.71 \mathrm{MA}$ discharge, the operational point in $a / L_{n}$ is very close to the location where the modes switch from the electron to the ion (ITG) direction, which is not observed in the $0.42 \mathrm{MA}$ case. It is expected that the ITG dominated modes, based on arguments of ohmic rotation reversals, would rotate in the counter-current direction, which is consistent with the LHCD results. For comparison, in the $0.42 \mathrm{MA}$ case, the dependence on density gradient is still strong, but there is no changeover to ion directed modes observed. While this drive mechanism is consistent in these simulations, it does not explain all of the rotation results observed with LHCD. Sub-dominant modes, which are not included, may play an important role and should be included for completeness, and rotation shear may become important in non-linear simulations as well.

The above discussions address the change in sign of the rotation, depending on the $q$ profile, but it remains to account for the original rotation before the $q$ profile evolution. The simplest explanation is that LH waves are Landau damped on passing electrons which collisionally transfer the momentum gained from the waves to the ions. The initial torque acting on the ions, as experimentally derived from the rate of change of the ion momentum, is generally consistent with this picture. However more subtle effects may be involved having to do with wave absorption on trapped and circulating electrons, and also the effect of the co-current part of the $k_{\|}$spectrum. When LH waves interact with trapped electrons in such a way that they would normally have contributed positively to the plasma current, the electrons are instead driven inward to conserve angular momentum [78]. This so-called rf-pinch effect must be quickly $(<\mathrm{ms})$ balanced by an inward ion flow to satisfy the ambipolarity constraint. The resulting Lorentz force acting on the ion flow gives rise to a counter-current torque. At low density and current, the counter-current torque due to wave absorption by trapped electrons is usually relatively weak, due to the low fraction of trapped electrons near the core and the high phase velocity of the waves. Passing electrons can also undergo a radial drift due to perpendicular momentum injection and this can lead to either a counter-current or co-current torque depending on whether the parallel index of refraction where the waves are absorbed, $n_{\| \text {abs }}$, is less than or greater than $n_{\phi}$, respectively [21]. However, the total torque acting on the ions is that transmitted by the waves radiated by the antenna, namely $R P n_{\phi} / c$, if all effects of frictional momentum transfer and momentum transfer due to the radial drift of passing and trapped electrons are accounted for. The radiated toroidal angular momentum cannot be lost and is fully transferred to ions on the ms time scale.

A challenge to this picture is to explain the change in rotation direction. Any such explanation based only on wave physics is likely to require that the absorbed spectrum must be co-directed. In fact, whenever LH waves are unidirectionally launched so as to accomplish the current drive effect at low $n_{\|}$, there is some power launched in the opposite toroidal 
direction at high $n_{\|}$. The power at high $n_{\|}$carries relatively more momentum, by the ratio of the wave number magnitudes; however, the launched power is approximately proportional to $1 / n_{\|}$with the result that the momentum carried by waves in the co-current toroidal direction is typically only $20-30 \%$ of that launched in the counter-current direction. Nevertheless if the high $n_{\|}$momentum were absorbed, it would contribute to a co-directed torque. Observe, however, that high $n_{\|}$waves have difficulty penetrating the plasma, whereas low $n_{\|}$waves more easily penetrate. In other words, the high $n_{\|}$waves, if absorbed at all, are absorbed peripherally to the low $n_{\|}$waves. Thus, in plasmas where the low $n_{\|}$waves penetrate the plasma core, the high $n_{\|}$waves might be absorbed only in the periphery. Moreover, in plasmas where the low $n_{\|}$waves cannot penetrate the plasma core, but are instead absorbed only in the periphery, the high $n_{\|}$waves might not be absorbed beyond the separatrix, but instead remain in the edge of the plasma within the scapeoff layer.

Note also that while there is speculation that the ubiquitous high $n_{\|}$power travelling in the unintended direction might play a role in plasma rotation, this power plays a negligible role in the LHCD effect [32]. That is because any momentum put into passing electrons by this part of the spectrum is absorbed by the slower electrons, which quickly share their momentum with the ions, thus extinguishing any current drive role for this part of the spectrum. While quantitatively unlikely in the scenarios described above, it is nevertheless in principle possible for the co-directed waves to dominate the torque balance while the counter-directed waves determine the direction of driven current.

There is strong evidence that for the high density $(>1.2 \times$ $10^{20} \mathrm{~m}^{-3}$ ) cases shown in figures $16-23$, the changes in the rotation are due to changes in transport caused by the $\mathrm{LH}$ waves. For low density target plasmas with good core LH wave absorption, the results are consistent with the qualitative explanation. The injected LH waves carry counter-directed momentum which is Landau absorbed, mainly by passing electrons, and which is transferred by collisions to the ions, resulting in a counter-directed torque. In general, $n_{\| \text {abs }}>n_{\phi}$, and wave momentum conservation then results in radial flows which lead to a $v_{r} \times B_{\text {pol }}$ toroidal force acting on the ions [21]. The rotation change represents a balance between the applied torque and the effective ion viscosity. However, if the LHCD power level is high enough to drive significant current (to decrease the core $\hat{s}$ or raise $q_{0}$ above 1 ), the sign of the residual stress can change, causing the rotation increment to switch to the co-direction. The last 2 rows in table 2 summarize the rotation drive agent and appropriate component of the momentum flux. Regardless of the mechanism details, LHRF wave injection may be a powerful tool for both co- and counterrotation profile control.

\section{Conclusions}

In summary, LH waves have been introduced into L- and $\mathrm{H}$-mode target plasmas, resulting in velocity changes in both the co- and counter-current directions. For low density L-mode plasmas, the rotation direction depends on the resulting changes in the $q$ profile, with co-rotation increments only seen in plasmas with $q_{0}>1$ (columns 1 and 2 in table 2, small asterisks and dots in figure 24). In these low density cases, the rotation changes are in the plasma core only, inside of $r / a=0.5$, and evolve on a time scale similar to the current relaxation time. For high density L-mode target plasmas with poor core $\mathrm{LH}$ wave absorption, the rotation changes are in the co-current direction, are seen over the entire profile, evolve on the shorter heat and momentum transport time scales and are caused by edge perturbations (column 3 in table 2, dots with ' + ' signs in figure 24 ). In high density H-mode plasmas, both co- and counter-rotation increments with LHRF are seen. For the co-current rotation increment cases, the edge absorption of LH waves leads to a steepening of the pedestal temperature gradient, which drives co-current rotation in the core, on a transport time scale (column 4 in table 2, large dots in figure 24). For the counter-increment examples, the edge absorption of the LH waves leads to a density pedestal reduction, which steepens the core density gradient, causing a suppression of the momentum pinch, which leads to a flattening of the core velocity profile (columns 5, 6 and 7 in table 2, large asterisks in figure 24). These examples exhibit the rich variety of ways that $\mathrm{LH}$ waves can lead to changes in the momentum flux.

\section{Acknowledgments}

The authors thank C. Fenzi, B. Chouli, F. Nave, M. Yoshida, S. Koide and Y. Shi for information regarding LHCD rotation and the Alcator C-Mod operations, LH and ICRF groups for expert running of the tokamak. Work supported at MIT by DoE Contract No DE-FC02-99ER54512 and in part by an appointment to the US DOE Fusion Energy Postdoctoral Research Programme administered by ORISE.

\section{References}

[1] Rice J.E. et al 2007 Nucl. Fusion 471618

[2] Fisch N.J. 1987 Rev. Mod Phys. 59175

[3] Lin Y. et al 2008 Phys. Rev. Lett. 101235002

[4] Lin Y. et al 2009 Phys. Plasmas 16056102

[5] Lin Y. et al 2011 Nucl. Fusion 51063002

[6] Lin Y. et al 2012 Plasma Phys. Control. Fusion 54074001

[7] Noterdaeme J.-M. et al 2003 Nucl. Fusion 43274

[8] Eriksson L.-G. et al 2009 Plasma Phys. Control. Fusion 51044008

[9] deGrassie J.S. et al 2004 Phys. Plasmas 114323

[10] Yoshida M. et al 2009 Phys. Rev. Lett. 103065003

[11] McDermott R.M. et al 2011 Plasma Phys. Control. Fusion 53035007

[12] Koide Y. et al 1993 Proc. 14th Int. Conf. on Plasma Physics and Controlled Nuclear Fusion Research 1992 (Wurzburg, Germany, 1992) vol 1 p 777 (Vienna: IAEA)

[13] Platz P. et al 1995 22nd European Physical Society Conf. on Plasma Physics and Controlled Fusion (Bournemouth, UK, July 1995) vol 19C Part III-337

[14] Sakamoto Y. et al 2006 Plasma Phys. Control. Fusion 48 A63

[15] Ince-Cushman A. et al 2009 Phys. Rev. Lett. 102035002

[16] Rice J.E. et al 2009 Nucl. Fusion 49025004

[17] Yuejiang S. et al 2011 Phys. Rev. Lett. 106235001

[18] Podpaly Y.A. 2012 Rotation Generation and Transport in Tokamak Plasmas PhD Thesis Massachusetts Institute of Technology

[19] Lee J. et al 2011 Proc. 19th Topical Conf. on Radio Frequency Power in Plasmas (Brussels, July 2010) vol 1406 p 459

[20] Gao Z. et al 2011 Phys. Plasmas 18082507

[21] Lee J. et al 2012 Plasma Phys. Control. Fusion 54125005 
[22] Xiaoyin G. et al 2013 Phys. Plasmas 20022502

[23] Hughes J.W. et al 2010 Nucl. Fusion 50064001

[24] Marmar E.S. et al 2007 Fusion Sci. Technol. 51261

[25] Basse N.P. et al 2007 Fusion Sci. Technol. 51476

[26] Lao L.L. et al 1985 Nucl. Fusion 251611

[27] Ko J. et al 2010 Rev. Sci. Instrum. 81033505

[28] Shiraiwa S. et al 2011 Phys. Plasmas 18080705

[29] Ince-Cushman A. et al 2008 Rev. Sci. Instrum. 7910 E302

[30] Reinke M.L. et al 2012 Rev. Sci. Instrum. 83113504

[31] Bonoli P.T. et al 2007 Fusion Sci. Technol. 51401

[32] Fisch N.J. 1978 Phys. Rev. Lett. 41873

[33] Schmidt A.E. et al 2011 Phys. Plasmas 18056122

[34] Wallace G.M. et al 2012 Phys. Plasmas 19062505

[35] Baek S.G. et al 2013 Plasma Phys. Control. Fusion 55052001

[36] Wallace G.M. et al 2013 Nucl. Fusion 53073012

[37] Shiraiwa S. et al 2013 Progress toward steady-state regimes in alcator C-Mod Nucl. Fusion submitted

[38] Garofalo A.M. et al 2008 Phys. Rev. Lett. 101195005

[39] Solomon W.M. et al 2009 Nucl. Fusion 49085005

[40] Rice J.E. et al 2011 Nucl. Fusion 51083005

[41] Rice J.E. et al 2011 Phys. Rev. Lett. 107265001

[42] Rice J.E. et al 2012 Phys. Plasmas 19056106

[43] Rice J.E. et al 2005 Nucl. Fusion 45251

[44] Bortolon A. et al 2006 Phys. Rev. Lett. 97235003

[45] Duval B.P. et al 2007 Plasma Phys. Control. Fusion 49 B195

[46] Rice J.E. et al 2008 Plasma Phys. Control. Fusion 50124042

[47] Duval B.P. et al 2008 Phys. Plasmas 15056113

[48] Rice J.E. et al 2013 Nucl. Fusion 53033004

[49] LaBombard B. et al 2004 Nucl. Fusion 441047

[50] Camenen Y. et al 2010 Plasma Phys. Control. Fusion 52124037

[51] Diamond P.H. et al 2009 Nucl. Fusion 49045002

[52] Gürcan Ö.D. et al 2007 Phys. Plasmas 14042306

[53] Kosuga Y. et al 2010 Phys. Plasmas 17102313
[54] Gürcan Ö.D. et al 2010 Phys. Plasmas 17112309

[55] Rice J.E. et al 2011 Phys. Rev. Lett. 106215001

[56] Lee W.D. et al 2003 Phys. Rev. Lett. 91205003

[57] Rice J.E. et al 2004 Nucl. Fusion 44379

[58] Buttery R.J. et al 1999 Nucl. Fusion 391827

[59] Wolfe S. et al 2005 Phys. Plasmas 12056110

[60] Golant V.E. 1972 Sov. Phys._Tech. Phys. 161980

[61] Greenwald M. et al 2007 Fusion Sci. Technol. 51266

[62] Lau C. et al 2012 Rev. Sci. Instrum. 83 10E309

[63] Hahm T.S. et al 2007 Phys. Plasmas 14072302

[64] Peeters A. et al 2007 Phys. Rev. Lett. 98265003

[65] Yoshida M. et al 2009 Nucl. Fusion 49115028

[66] deVries P.C. et al 2010 Plasma Phys. Control. Fusion 52065004

[67] Tala T. et al 2011 Nucl. Fusion 51123002

[68] McDermott R.M. et al 2011 Plasma Phys. Control. Fusion 53124013

[69] Angioni C. et al 2011 Phys. Rev. Lett. 107215003

[70] Weisen H. et al 2012 Nucl. Fusion 52042001

[71] Smirnov A.P. and Harvey R. 1995 Bull. Am. Phys. Soc. 401837

[72] Harvey R.W. and McCoy M. 1992 Proc. of the IAEA TCM on Simulation and Modeling of Thermonuclear Plasmas (Montreal, Canada, 1992) p 489

[73] Wang L. and Diamond P.H. 2013 Gryokinetic theory of turbulent acceleration of parallel rotation in tokamak plasmas Phys. Rev. Lett. submitted

[74] Kwon J.M. et al 2012 Nucl. Fusion 52013004

[75] Yi S. et al 2012 Phys. Plasmas 19112506

[76] Yi S. et al 2013 Implications of non-resonant convective cells for turbulence intensity profile, shear flow and transport Nucl. Fusion submitted

[77] Kotschenreuther M. et al 1995 Comput. Phys. Commun. 88128

[78] Fisch N.J. and Karney C.F.F. 1981 Phys. Fluids 2427 